\title{
A biallelic variant in CLRN2 causes non-syndromic hearing loss in humans
}

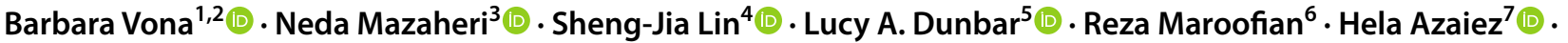

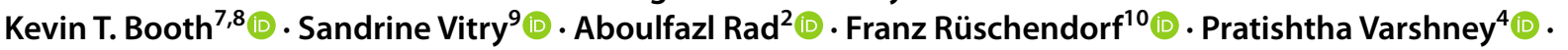 \\ Ben Fowler ${ }^{11}$. Christian Beetz ${ }^{12}$ - Kumar N. Alagramam ${ }^{13,14,15} \cdot$ David Murphy $^{6}$ (1) Gholamreza Shariati ${ }^{16,17}$.

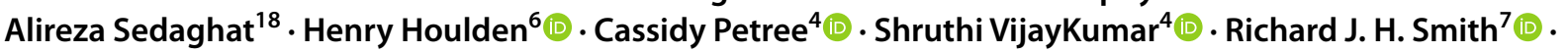

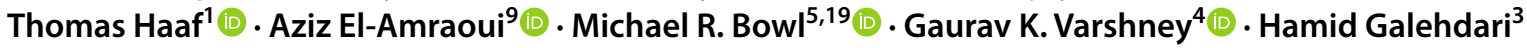

Received: 25 September 2020 / Accepted: 31 December 2020 / Published online: 26 January 2021

(c) The Author(s) 2021

\begin{abstract}
Deafness, the most frequent sensory deficit in humans, is extremely heterogeneous with hundreds of genes involved. Clini$\mathrm{cal}$ and genetic analyses of an extended consanguineous family with pre-lingual, moderate-to-profound autosomal recessive sensorineural hearing loss, allowed us to identify $C L R N 2$, encoding a tetraspan protein, as a new deafness gene. Homozygosity mapping followed by exome sequencing identified a $14.96 \mathrm{Mb}$ locus on chromosome 4p15.32p15.1 containing a likely pathogenic missense variant in CLRN2 (c.494C > A, NM_001079827.2) segregating with the disease. Using in vitro RNA splicing analysis, we show that the CLRN2 c.494C > A variant leads to two events: (1) the substitution of a highly conserved threonine (uncharged amino acid) to lysine (charged amino acid) at position 165, p.(Thr165Lys), and (2) aberrant splicing, with the retention of intron 2 resulting in a stop codon after 26 additional amino acids, p.(Gly146Lysfs*26). Expression studies and phenotyping of newly produced zebrafish and mouse models deficient for clarin 2 further confirm that clarin 2 , expressed in the inner ear hair cells, is essential for normal organization and maintenance of the auditory hair bundles, and for hearing function. Together, our findings identify CLRN2 as a new deafness gene, which will impact future diagnosis and treatment for deaf patients.
\end{abstract}

\section{Introduction}

The mammalian inner ear is an exquisite and highly complex organ, made up of the vestibule, the organ responsible for balance, and the cochlea, the sensory organ for hearing. The

Neda Mazaheri, Sheng-Jia Lin, Lucy A. Dunbar contributed equally.

Aziz El-Amraoui, Michael R. Bowl, Gaurav K. Varshney and Hamid Galehdari share senior authorship.

Supplementary Information The online version contains supplementary material available at https://doi.org/10.1007/s0043 9-020-02254-z.

Barbara Vona

barbara.vona@uni-wuerzburg.de;

barbara.vona@uni-tuebingen.de

Michael R. Bowl

m.bowl@har.mrc.ac.uk; m.bowl@ucl.ac.uk

Extended author information available on the last page of the article auditory sensory cells of the inner ear are called the inner and outer hair cells that are responsible for transduction of sound wave-induced mechanical energy into neuronal signals (Gillespie and Müller 2009; Hudspeth 1997). The functional mechanoelectrical transduction machinery involves intact formation and maintenance of a highly specialized and organized structure, the hair bundle. The hair bundle contains a few dozen F-actin-filled stereocilia, arranged in a highly interconnected and highly organized staircase-like pattern, which is critical for function (Kazmierczak et al. 2007). Knowledge of the mechanisms of formation, maintenance, and function of the transduction complex is limited (Cunningham and Müller 2019). In this regard, identification of novel genes that encode protein products essential for hearing is likely to improve our understanding of the physical, morphological and molecular properties of hair cells and associated mechanistic processes.

Hereditary hearing loss is one of the most common and genetically heterogeneous disorders in humans (Wright et al. 2018). Sensorineural hearing loss has an incidence of 1 to 
2 per 1000 at birth (Morton and Nance 2006). It displays extraordinary phenotypic, genetic and allelic heterogeneity, with up to 1000 different genes potentially involved (Ingham et al. 2019). So far, about 120 genes and more than 6000 disease causing variants (Azaiez et al. 2018) have been identified as responsible for non-syndromic hearing loss in humans (see http://hereditaryhearingloss.org/ and http:// deafnessvariationdatabase.org/), and many more are yet to be discovered. Genetic factors predominate the etiological spectrum and most hereditary hearing loss appears to follow an autosomal recessive inheritance pattern (Smith et al. 2005). To date, approximately $80 \%$ of the known autosomal recessive deafness-associated genes have been originally identified by studying extended consanguineous families (Hofrichter et al. 2018). There are many forms of hearing loss that are clinically indistinguishable but caused by distinct genetic entities that are presently unknown. Identification of additional genes essential for auditory function, through the study of families exhibiting hereditary hearing loss, will not only help increase our understanding of the biology of hearing, but will also identify new molecular targets for therapeutic intervention.

Through the study of an extended consanguineous Iranian family, we have identified a CLRN2 coding lesion as the likely cause of hearing loss in family members that are homozygous for the allele. We have established that clarin 2 likely plays a critical role in mechanotransducing stereocilia of the hair bundle in zebrafish and mouse. CLRN2 belongs to the clarin (CLRN) family of proteins that are comprised of three orthologues named clarin 1,2, and 3 that encode four-transmembrane domain proteins. Pathogenic variants in CLRN1 (clarin 1) cause either non-syndromic retinitis pigmentosa (RP) (Khan et al. 2011) or Usher syndrome type 3A (USH3A), that is characterized by progressive hearing loss, RP and variable vestibular dysfunction (Adato et al. 2002; Joensuu et al. 2001; Ness et al. 2003; Plantinga et al. 2005). This study establishes clarin 2 as essential for inner ear function in zebrafish, mice and humans, with a loss-of-function allele leading to autosomal recessive non-syndromic sensorineural hearing loss (ARNSHL).

\section{Materials and methods}

\section{Patient clinical and audiometry data}

Written informed consent was provided from all participating individuals. This study has been approved by the Faculty of Medicine ethics commissions at the University of Würzburg (46/15) and Shahid Chamran University of Ahvaz (\#EE/97.24.3 17654). A three generation Iranian family of Lurs ethnicity was ascertained as part of a large ethnically diverse Iranian population rare disease study. Pure-tone audiograms and medical information were collected from participating members. Clinical examination excluded additional syndromic features.

Individuals IV-1, IV-6, and V-1 (Fig. 1) underwent complete ear, nose and throat examination, including binocular ear microscopy and external ear inspection. Routine puretone audiometry was performed according to current standards that measured hearing thresholds at frequencies 0.25 , $0.5,1,2,4,6$ and $8 \mathrm{kHz}$. Both air- and bone-conduction thresholds were determined. Severity of hearing loss was defined as previously described (Mazzoli et al. 2003). Individuals IV-1 and IV-6 underwent additional tympanometry and speech recognition threshold testing. Audiometry testing for individuals IV-1, IV-6, and V-1 was performed at ages 29,44 , and 20 years, respectively.

\section{Genotyping, gene mapping, copy number variation and exome sequencing data analyses}

Due to parental consanguinity and suspected autosomal recessive mode of inheritance, we assumed that the causal variant would be homozygous and identical by descent in affected individuals in the fourth generation of the family. Blood samples from 14 family members were obtained and genomic DNA was isolated from whole blood using standard procedures. DNA from affected (IV-1, IV-6, and IV-8) and unaffected (IV-2, IV-3, IV-4, and IV-5) individuals were genotyped using the Infinium Global Screening Array-24 v1.0 BeadChip (Illumina, San Diego, CA, USA) according to manufacturer's protocols. Copy number variation calling was performed using GenomeStudio v.2011.1 and cnvPartition 3.2.0 (Illumina).

From the 618,540 markers on the array, we filtered out InDels, MT- and Y-chromosomal SNPs, multi-allelic SNPs, SNPs with missing genotypes in more than one individual, and SNPs having a minor allele frequency (MAF) lower than $5 \%$ in gnomAD European individuals (NFE) resulting in 242,705 bi-allelic SNPs for quality control (QC) and Linkage analysis. Data conversion to Linkage format files and QC was managed with the ALOHOMORA software (Rüschendorf and Nürnberg 2005). The sex of individuals was estimated by counting heterozygous genotypes on the $\mathrm{X}$-chromosome and compared to the given pedigree data. The relationships between family members were verified with the program Graphical Relationship Representation (GRR) (Abecasis et al. 2001). PedCheck (O'Connell and Weeks 1998) was used to detect Mendelian errors (ME) and SNPs with ME were removed from the data set. Unlikely genotypes, e.g., double recombinants, were identified with Merlin (Abecasis et al. 2002) and deleted in the individuals.

Linkage analysis was performed with Merlin (Abecasis et al. 2002) using an autosomal recessive mode of 
A Iranian consanguineous family

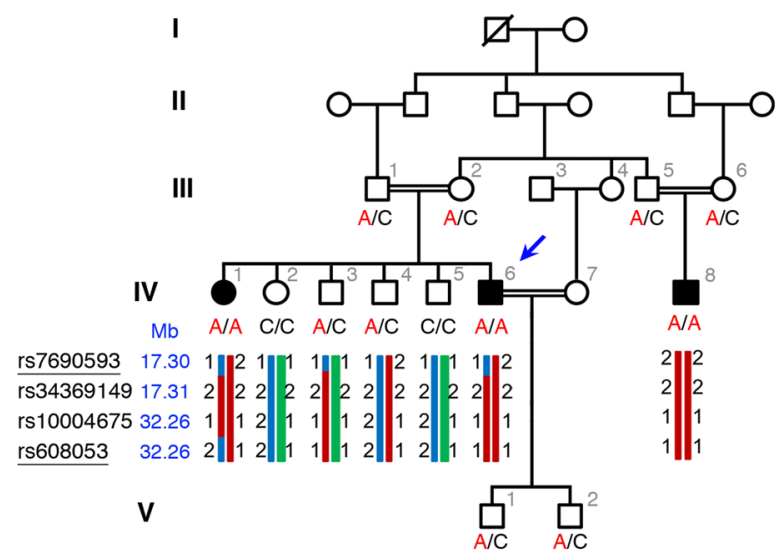

B Pure-tone audiograms of a normal hearing ( $\mathrm{V}-1 / 20$ years, -$)$ and CLRN2 affected (IV-1/29 years, - \& IV-6/44 years, - ) individuals
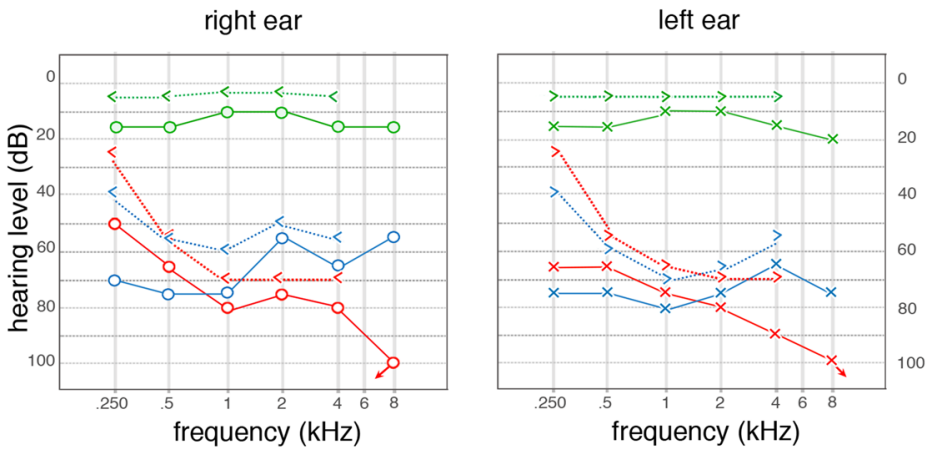

Air conduction thresholds ( $\equiv$ ) Bone conduction thresholds (::*:::: )

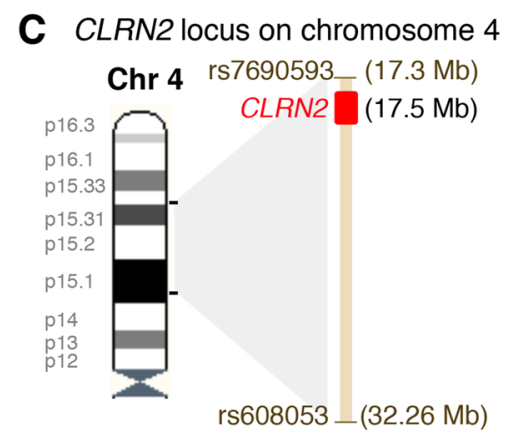

D Electropherograms of normal (c.494C) and CLRN2 (c.494A) variants

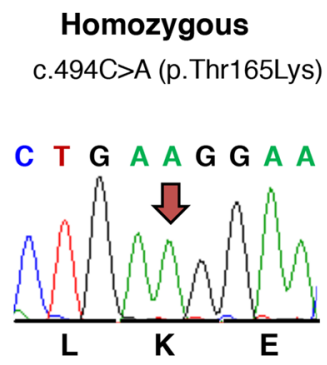

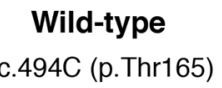

C T G A C G G A A

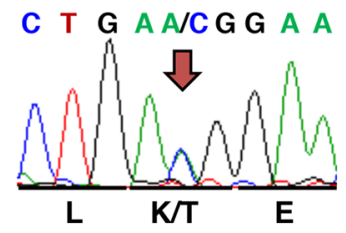

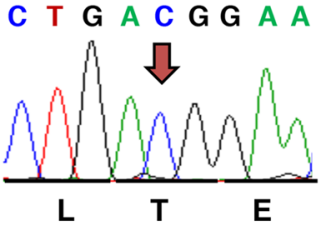

Fig. 1 Pedigree, audiological data, genetic data, and locus mapping. a The consanguineous family of Iranian origin with hearing loss and segregation of the CLRN2 c. $494 \mathrm{C}>\mathrm{A}$ variant. Linked haplotypes harbouring the pathogenic variant coloured in red with meiotic recombination SNP markers underlined. SNP positions are annotated using the GRCh37 human genome assembly. b Pure-tone audiograms from affected individuals IV-1 (red) and IV-6 (blue), as well as an unaffected heterozygous individual V-1 (green). Air-conduction thresh-

inheritance with complete penetrance and a mutant allele frequency of 0.001. To avoid the problem of Linkage Disequilibrium (LD) between markers, which can lead to inflated LOD scores, we created a less dense, LD-reduced marker set of 91,426 SNPs with a minimal distance of 10,000 bases between markers and a MAF $>0.15$. The exact position of the LOD score regions, e.g., the recombination events, were identified with the full marker set of 242,705 SNPs.

Additionally, homozygosity mapping was performed using HomozygosityMapper to identify common homozygous intervals among the affected individuals (Seelow et al. 2009). Runs of homozygosity with a maximum threshold of 0.99 were checked after the exome-wide analysis was completed.

For exome sequencing, DNA samples from two affected individuals (IV-1 and IV-6) were used. The data from individual IV-6 were analyzed exome-wide and data from individual IV-1 were used for determination of allele sharing. Exome capture using genomic DNA was performed using olds in $\mathrm{dB}$ HL for the right and left ears are represented by circles and crosses, respectively. Bone-conduction thresholds are represented by $<$ and $>$ for right and left ears, respectively, and confirm a sensorineural hearing loss in the affected individuals. c Linkage mapping reveals a $14.96 \mathrm{Mb}$ locus on chromosome 4 containing CLRN2. d Sequence electropherograms showing the homozygous, heterozygous and WT images of the CLRN2 c.494C > A; pThr165Lys pathogenic variants

the SureSelect Target Enrichment v6 (Agilent) kit following manufacturer's recommendations. The libraries were sequenced with a HiSeq4000 (Illumina). Data analysis was performed using the Burrows-Wheeler Alignment (BWA) tool for read mapping to the human reference genome GRCh37 (hg19), Picard for duplicate removal, GATK for local re-alignment, base recalibration, variant calling, and variant annotation, and SnpEff for variant annotation. Variant filtering was based on: coverage $>10 \mathrm{X}$, Phred quality score $\geq 30$, and MAF $\leq 0.005$ as reported in 1000 Genomes Project and EVS6500. Variants were filtered based on coding effect (non-synonymous, synonymous, indels, and splice site variants), and artifact-prone genes (HLAs, MAGEs, $M U C \mathrm{~s}, N B P F \mathrm{~s}, O R \mathrm{~s}, P R A M E \mathrm{~s})$ were excluded. ACMG guidelines were used for variant interpretation (Oza et al. 2018). Visualization was performed using the Integrative Genomics Viewer. Analysis of homozygous and compound heterozygous variants between the two sequenced affected individuals (IV-6 and IV-1) followed. We analyzed missense 
variants using a combination of criteria that scored conservation using GERP++ and PhyloP, and deleterious or pathogenic scores in Combined Annotation Dependent Depletion (CADD) (Kircher et al. 2014), LRT (Chun and Fay 2009), MutationTaster (Schwarz et al. 2014), PolyPhen-2 (Adzhubei et al. 2010), and SIFT (Ng and Henikoff 2001). Missense variants were excluded when three out of five in silico pathogenicity prediction tools yielded a benign score. Manual MAF analysis used gnomAD (Lek et al. 2016), GME (Scott et al. 2016) and Iranome (Fattahi et al. 2019). Potential effects on splicing were assessed using ESEfinder (Cartegni et al. 2003) and RESCUE-ESE (Fairbrother et al. 2004).

\section{Segregation, sequence and in vitro splicing analyses of the CLRN2 c.494C > A likely pathogenic variant}

To confirm segregation of the CLRN2 c.494C > A; p.(Thr165Lys) (NM_001079827.2) homozygous variant, Sanger sequencing was completed in all 14 family members using the following primers (CLRN2 Ex3 F: 5'-AAATGC CACCTCTTACAGAGTTGC-3' and CLRN2 Ex3 R: 5'-ACC GTGGCCTCTTCGATTTTGGTC-3') and standard PCR and sequencing parameters.

To document residue conservation, CLRN1 (UniProt: P58418) and CLRN2 (UniProt: A0PK11) were aligned and visualized in Jalview (Waterhouse et al. 2009) with an overview of the pathogenic and likely pathogenic missense and nonsense CLRN1 variants retrieved from the Deafness Variation Database v 8.2 (Azaiez et al. 2018).

In addition, secondary protein structure prediction of human CLRN2 (NP_001073296.1) that included the wildtype (WT) and mutated amino acid residues was performed using I-TASSER (Yang et al. 2015).

To assess the splicing effect of the c.494C > A variant, in vitro splicing assays, also called mini-genes, were carried out as described (Booth et al. 2018a, b). WT CLRN2 exon 3 (266 bp) plus 183 and 51 nucleotides from intron 2 and the 3'UTR were PCR amplified with gene-specific primers containing Sall or SacII restriction enzyme sites, respectively. After PCR amplification, clean up, and restriction enzyme digestion, the PCR fragment was ligated into the pET01 Exontrap vector (MoBiTec) and the sequence was confirmed. Variants were then introduced into the WT sequence using QuikChange Lightning Site-Directed Mutagenesis (Agilent) according to the manufacturer's protocols via overlapping primers containing the alteration. The WT and mutant mini-genes were sequence confirmed.

WT or mutant mini-genes were transfected in triplicates into COS-7 and ARPE-19 cells using TransIT-LT1 Transfection Reagent (Mirus). Cells were harvested $36 \mathrm{~h}$ after transfection and total RNA was extracted using Quick-RNA MiniPrep Plus kit (ZYMO Research). cDNA was transcribed using 750 ng of isolated RNA SuperScript ${ }^{\mathrm{TM}}$ III Reverse Transcriptase (ThermoFisher Scientific) using a primer specific to the $3^{\prime}$ native exon of the pET01 vector according to manufacturer's protocol. PCR amplification followed using primers specific to the $5^{\prime}$ and $3^{\prime}$ native exons of the pET01 vector, and products were visualized on a $1.5 \%$ agarose gel. As a negative control, rs117875715 (chr4(GRCh37):g.17,5 $28,480 \mathrm{G}>\mathrm{A}$ ), a benign polymorphism, was used to test and validate the designed mini-gene assay.

Concurrently, the mini-gene splice assay experiment was conducted in a double-blind manner as previously described (Lekszas et al. 2020). Genomic DNAs of an affected homozygous (IV-6) and WT individual (IV-5) were amplified using a forward primer with a XhoI restriction site (CLRN2 Ex3 XhoI F: 5'-aattctcgagTTGCAGTGAGCTGAG ATGGT-3') and a reverse primer with a BamHI restriction site (CLRN2 Ex3 BamHI R: 5'-attggatccGCCTTGCGAAGT TGTTACTG-3'). The 886 bp amplicon included the entire exon 3 sequence plus additional flanking $320 \mathrm{bp}\left(5^{\prime}\right)$ and 306 bp ( $\left.3^{\prime}\right)$ sequence that was ligated into a multiple cloning site between native exons A and B in the linearized pSPL3b exon-trapping vector. The vector was transformed into DH5 $\alpha$ competent cells and plated overnight. All mutant mini-genes were Sanger sequence confirmed.

Homozygous and WT mini-genes were transfected in triplicate into HEK 293 T cells cultured in FCS-free medium in 6 well culture plates with a density of $2 \times 10^{5}$ cells per $\mathrm{mL}$. The mini-genes in the pSPL $3 b$ vector were transiently transfected using $6 \mu \mathrm{l}$ of FuGENE 6 Transfection Reagent (Roche) with $2 \mu \mathrm{g}$ of vector. An empty vector and HEK $293 \mathrm{~T}$ cells were included as controls. The transfected cells were harvested $24-48 \mathrm{~h}$ post-transfection. Total RNA was prepared using the miRNAeasy Mini Kit (Qiagen). Approximately, $1 \mu \mathrm{g}$ of RNA was reverse transcribed using a High Capacity RNA-to-cDNA Kit (Applied Biosystems) following manufacturer's protocols. The cDNA was used for PCR amplification using a vector specific SD6 forward (5'-TCT GAGTCACCTGGACAACC-3') and a terminal CLRN2 exon 3 reverse cDNA primer (5'-CAAGATATCCTCAGCTGT GACC-3'). The resulting amplified fragments were visualized on a $1.5 \%$ agarose gel. cDNA amplicons were Sanger sequenced. cDNA amplicons from the homozygous individual were cloned following standard protocols for the TA cloning (dual promoter with pCRII) kit (Invitrogen).

\section{CRISPR/Cas9-mediated inactivation of clrn2 in zebrafish}

Zebrafish (Danio rerio) were raised and maintained in an AALAC accredited facility at the Oklahoma Medical Research Foundation (OMRF) under standard conditions. Zebrafish embryos/larvae were maintained in embryo medium with $0.00002 \%$ methylene blue and raised at $28^{\circ} \mathrm{C}$. 
All animal experiments were performed as per protocol (1701) and approved by the Institutional Animal Care Committee of OMRF (IACUC). All zebrafish handling, embryo care, and microinjections were performed as previously described (Westerfield 2000). WT zebrafish strain NHGRI-1 was used for all experiments (LaFave et al. 2014). The zebrafish embryonic staging was determined by morphological features according to (Kimmel et al. 1995).

To produce zebrafish $c l r n 2$ crispants, the sgRNA target sequences were selected from the UCSC genome browser tracks generated by the Burgess lab. Five independent targets were chosen and sgRNAs were synthesized by in vitro transcription as described earlier (Varshney et al. 2016). sgRNAs and Cas9 protein complex were used to generate indels. A $6 \mu \mathrm{L}$ mixture containing $2 \mu \mathrm{L}$ of $40 \mu \mathrm{M}$ Spy Cas 9 NLS protein (New England Biolabs, MA, USA), $200 \mathrm{ng}$ each of five sgRNAs (in $2 \mu \mathrm{L}$ ) and $2 \mu \mathrm{L}$ of $1 \mathrm{M}$ potassium chloride was injected into one-cell-stage WT embryos. Injection volumes were calibrated to $1.4 \mathrm{~nL}$ per injection. Insertion/ deletion (indel) variants were detected by amplifying the target region by PCR and Sanger sequencing as described earlier (Varshney et al. 2016). The sequencing data were analyzed by Inference of CRISPR Edits (ICE) v2 CRISPR analysis tool. The sgRNA target sequences and PCR primer sequences are listed in Supplementary Table S1.

\section{Zebrafish RNA extraction and quantitative reverse transcription PCR (RT-qPCR)}

Total RNA at different developmental stages, adult tissues, and CRISPR/Cas9 injected larvae were extracted using the TRIzol Reagent (Thermo Fisher Scientific, CA, USA) and purified by RNA clean and concentrator-5 kit (Zymo Research, CA, USA) according to the manufacturer's instructions. RNA concentration was measured by DeNovix DS-11 spectrophotometer (DeNovix Inc., USA). The cDNA was synthesized by iScript RT Supermix (Bio-Rad, USA), and was used as a template for performing the RT-qPCR with SYBR Green Supermix (Thermo Fisher Scientific, CA, USA) and the Light Cycler® 96 System (Roche, CA, USA). All RT-qPCR reactions were carried out using three biological and technical replicates. The housekeeping gene $18 S$ was used as a reference gene.

All RT-qPCR primer pairs were designed across exonexon junctions using NCBI Primer-BLAST program (Supplementary Table S1). PCR cycling conditions were used as per manufacturer instructions. All reactions were carried out using three biological and technical replicates. The housekeeping gene $18 S$ was used as a reference gene. Amplification specificity was assessed by dissociation curve analysis. The cycle threshold values $(\mathrm{Ct})$ data were imported into Microsoft Excel for the relative gene expression analysis. Quantification was based on $2^{\wedge}(-\Delta \Delta \mathrm{CT})$ method (Livak and
Schmittgen 2001), and using $18 \mathrm{~h}$ post fertilization (hpf) for clarin 2 temporal expression, muscle for clarin 2 in different tissue expression and the corresponding age-matched control for clarin 2 CRISPR injected $\mathrm{F}_{0}$ larvae as normalization control.

\section{Distribution of clrn2, phalloidin staining and behavioral analysis in zebrafish}

To determine $\operatorname{clrn} 2$ expression, we used in situ hybridization on larvae and inner ear-containing cryosections. The full-length coding sequence of zebrafish clarin 2 (NM_001114690.1) was PCR amplified from WT zebrafish cDNA using primer pairs with Bam $\mathrm{HI}$ and XhoI restriction sites cloned into the pCS2+ vector (a kind gift from Dr. Dave Turner, University of Michigan). After restriction digestion, the resulting clones were sequenced and used as templates for riboprobe synthesis. The digoxigenin-UTP-labeled riboprobes were synthesized according to the manufacturer's instructions (Millipore Sigma, MO, USA). Briefly, the clarin 2 and the pvalb9 plasmids (Horizon Discovery) were linearized by BamHI and NotI restriction enzymes, respectively. The linearized plasmid was purified and used as template for in vitro transcription using T7 RNA polymerase to synthesize anti-sense probes. The sense probe was made using $\mathrm{XbaI}$ linearized clarin 2 plasmid and SP6 RNA polymerase.

Whole-mount in situ hybridization (WISH) on 3 and 5 dpf zebrafish embryos/larvae was performed following the procedures as described by Thisse et al. with minor modifications (Thisse and Thisse 2008). Age-matched zebrafish embryos were randomly collected by breeding WT zebrafish pairs. The embryos were treated with $0.003 \%$ phenylthiourea (PTU) (Millipore Sigma, MO, USA) in embryo medium at 1 day post-fertilization (dpf) until the desired stages reached to reduce the pigment formation that will facilitate color visualization during in situ hybridization. Embryos/larvae were then fixed with $4 \%(\mathrm{~V} / \mathrm{V})$ paraformaldehyde in phosphate-buffered saline (PBS) at 3 and $5 \mathrm{dpf}$. An additional bleaching step was carried out after fixation by incubating the embryos at room temperature in a $3 \%$ hydrogen peroxide and $0.5 \%$ potassium hydroxide solution. The permeabilization of $3 \mathrm{dpf}$ embryos and $5 \mathrm{dpf}$ larvae was performed using $2 \mu \mathrm{g} / \mathrm{mL}$ proteinase $\mathrm{K}$ for 12 and $18 \mathrm{~min}$, respectively. Color development was conducted using the BM-Purple alkaline phosphatase substrate (Millipore Sigma, MO, USA).

For preparation of cryo-sectioned samples after WISH, the 5 dpf larvae were soaked in 25, 30\% (V/V) sucrose/PBS and optimum cutting temperature (OCT) each for at least 2 days, and embedded in OCT, then Cryotome sectioned at a $10-\mu \mathrm{m}$ thickness.

For phalloidin staining of the zebrafish inner ear, $5 \mathrm{dpf}$ larvae were euthanized with tricaine and fixed in $4 \%$ (V/V) paraformaldehyde (PFA) at $5 \mathrm{dpf}$, fixed embryos were 
washed by PBSTx (1\% PBS, $0.2 \%$ triton X-100) and incubated in $2 \%$ triton X-100 in PBS at room temperature for overnight with agitation until the otoliths were completely dissolved. The larvae were sequentially washed in PBSTx and incubated with Alexa Fluor 488 Phalloidin (1:50) (Thermo Fisher Scientific, CA, USA) in PBSTw (1\% PBS, $0.1 \%$ Tween-20) at room temperature for $4 \mathrm{~h}$. The samples were washed in PBSTx after staining and mounted laterally in $75 \%$ glycerol on slides. Images were acquired with a Zeiss LSM-710 Confocal microscope.

To perform the acoustic evoked behavioral response (AEBR) test, $6 \mathrm{dpf}$ larvae were placed in a 48 -well plate with $200 \mu \mathrm{L}$ embryo water and placed in a Zebrabox (ViewPoint Life Sciences) and embryos were adapted in the dark for 15-30 min (until spontaneous movements were less frequent). The embryos were subjected to a $100 \mathrm{~ms}, 1 \mathrm{kHz}$ pure tone at $100 \%$ target power every $20 \mathrm{~s}$ for $4 \mathrm{~min}$ (12 stimuli) in the dark. The Zebrabox recorded the animals using infrared light and measured the activity as pixel changes over time. The burst threshold was set at 50 pixels, the freeze threshold set at 10 pixels, and sensitivity was set at 20 pixels. Movement over the 50 pixel burst threshold within the $2 \mathrm{~s}$ after stimulus was considered an evoked response. Responses were excluded if the larvae had spontaneous movement within the $2 \mathrm{~s}$ before the stimulus. All responses for a larva were excluded if they had spontaneous movement before 6 or more of the stimuli. The response rate was calculated by how many times an embryo had an evoked response out of the total number of stimuli and converted to a percentage.

\section{Production and phenotyping of clarin 2 deficient mutant in mice}

The Clrn $2^{\text {del629 }}$ mutant line was generated on a C57BL/6N background by the Molecular and Cellular Biology group at the Mary Lyon Centre (MLC), MRC Harwell Institute, using CRISPR/Cas9 genome editing (Dunbar et al. 2019). The mice were housed and maintained under specific pathogen-free conditions in individually ventilated cages, with environmental conditions as outlined in the Home Office Code of Practice. Animals were housed with littermates until weaned, and then housed with mice of the same sex and of similar ages, which was often their littermates. Both male and female animals were used for all experiments. Animal procedures at the MRC Harwell Institute were licenced by the Home Office under the Animals (Scientific Procedures) Act 1986, UK and additionally approved by the Institutional Animal Welfare and Ethical Review Body (AWERB). The ClrnI ${ }^{-/-}$mice (ClrnI ${ }^{\text {tm1.2Ugpa }}$, MGI: 6099052) used for comparative scanning electron microscopy analyses were previously described (Dulon et al. 2018).

To screen mice for auditory phenotypes and investigate auditory function, Auditory Brainstem Response (ABR) tests (measured using a click stimulus and frequency-specific tone-burst stimuli (at 8, 16 and $32 \mathrm{kHz}$ ) and Distortion Product Oto-Acoustic Emission (DPOAE) tests (measured using frequency-specific tone-burst stimuli from 8 to $32 \mathrm{kHz}$ with the TDT RZ6 System 3 hardware and BioSig RZ software (Tucker Davis Technology, Alachua, FL, USA)) were performed as described in Dunbar et al., 2019. For scanning electron microscopy imaging, fixed inner ear samples were processed by the osmium tetroxide/thiocarbohydrazide (OTOTO) method, as previously described (Dulon et al. 2018; Dunbar et al. 2019). Samples were visualized with a JSM-6010LV Scanning Electron Microscope (JEOL). Micrographs were pseudo-coloured in Adobe Photoshop.

\section{Statistical analysis}

To compare the $c l r n 2$ mRNA expression in zebrafish, data are presented as mean values \pm standard deviation (SD). Statistical analysis was performed using GraphPad Prism version 8.4 (GraphPad Software, San Diego, CA, USA). The significance level was set to 0.05 . The $\mathrm{p}$ value was determined using a two-tailed unpaired Student's t-test for RTqPCR of clrn 2 mRNA expression, and a two-tailed unpaired nonparametric Mann-Whitney U test for AEBR analysis. The statistical significance is represented in the figures as $* * * p<0.001$.

To assess ABR thresholds and DPOAE responses in the Clrn $2^{\text {del629 }}$ mice, one-way ANOVA statistical tests were used. Each frequency was tested for statistical significance separately. A threshold of $p>0.05$ was used to determine if differences were statistically significant. Statistical significance is represented in the figure as follows: $*^{* *} p<0.001$. All data shown are mean $\pm \mathrm{SD}$, and all statistical analyses was performed in GraphPad Prism.

\section{Results}

\section{Identification of CLRN2 as a novel deafness gene in a consanguineous Iranian family exhibiting autosomal recessive non-syndromic sensorineural hearing loss}

A three generation Iranian family of Lurs ethnicity was ascertained as part of a large ethnically diverse Iranian population rare disease study (Fig. 1a). Three individuals that included the proband (IV-6), his sibling (IV-1), and a cousin (IV-8), born form consanguineous marriages, have reported moderate-to-profound bilateral non-syndromic sensorineural hearing loss (Fig. 1b). The age of onset for these three individuals was between 2 and 3 years of age. Pure-tone air- and bone-conduction audiometry thresholds (Fig. 1b) show evidence of intrafamilial variability. Individual IV-1 
has a down sloping audiogram, with bilateral moderate-toprofound deafness. Individual IV-6 presented a moderateto-severe hearing loss with slightly better hearing at higher frequencies. Both individuals showed normal (type A) tympanograms bilaterally. Speech recognition thresholds for individual IV-1 were 80 and $75 \mathrm{~dB}$ at 84 and $88 \%$ for right and left ears, respectively, and a most comfortable level of $95 \mathrm{~dB}$. Speech recognition thresholds for individual IV-6 were 75 and $80 \mathrm{~dB}$, each at $84 \%$, for right and left ears, respectively. Patients have normal neuromotor, speech and language development, and did not show signs of impaired balance. No other abnormalities, including potential vision deficit, were present in the affected individuals, who were last evaluated at the age of 29 (IV-1), 44 (IV-6), and 25 (IV8 ) years. For comparison, pure-tone audiometry was also recorded from a family member (V-1), with no reported history of hearing deficits.

To identify the underlying genetic lesion, genome-wide linkage analysis identified one single significant maximum LOD score of 3.8 on chromosome 4 (Supplementary Fig. S1a-b). Linkage analysis and haplotyping with the dense marker set showed the adjacent markers rs7690593 and rs608053 (GRCh37/hg19, chr4: 17,298,007-32,261,222 bp, hg19). The homozygous region spans $14.96 \mathrm{Mb}$ and overlaps with the CLRN2 gene position (Fig. 1c). As a second independent method, we applied homozygosity mapping in the extended family to identify a $15.2 \mathrm{Mb}$ locus on chromosome 4p15.32p15.1 (GRCh37/hg19, chr4:17,298,445$32,495,165)$, defined by the SNPs rs7692897 and rs 17081424 (Supplementary Fig. S1c, Supplementary Table S2) that mirror the linkage results. This locus contains 30 genes, none of which are presently associated with deafness in humans (Supplementary Table S2). This approach also revealed four much smaller homozygous intervals on chromosomes 2p21 (137.3 kb), 3p22.2 (262.5 kb), 13q13.1 (90.7 kb), and 17q21.31 (292.6 kb) (Supplementary Fig. S1c, Supplementary Table S2) that do not contain known deafness-associated genes (Supplementary Table S2). Pathogenic copy number variations were excluded. Next, we undertook exome sequencing of affected individual IV-6 (arrow, Fig. 1a). This generated 56,387,543 mappable reads, with $75.5 \%$ on-target reads. The mean depth was 57.3-fold, with $97.3 \%$ of regions with a tenfold read depth. Analysis of the exome data of individual IV-6 excluded any candidate pathogenic variants in known deafness-associated genes (Doll et al. 2020) prompting an exome-wide analysis followed by filtering and re-analysis of variants in homozygous intervals (Supplementary Table S3). Further, close inspection of the exome sequencing data revealed complete sequencing coverage of genes in the homozygous intervals (Supplementary Table S4). Variant filtering detected a single homozygous missense variant in CLRN2 c.494C > A, (p.(Thr165Lys)) (NM_001079827.2) in the linked and homozygous interval on chromosome 4 (Supplementary Fig. S1a-c). This variant was shared with individual IV-1 and segregated in the extended family comprising a total of 14 individuals (Fig. 1a, d) and was the only remaining variant in the locus fulfilling variant filtering criteria. Only individuals homozygous for the CLRN2 c. $494 \mathrm{C}>\mathrm{A}$ variant exhibit hearing loss confirming the recessive nature of the allele (Fig. 1a). This variant fulfills ACMG criteria for classification as likely pathogenic (PM2_Moderate, PP1_Strong, PP3_Supporting).

\section{The CLRN2 c.494C > A leads to a likely pathogenic missense substitution and aberrant splicing}

The c.494C > A variant on chromosome 4p15.32 is unanimously predicted to be deleterious and disease causing by in silico tools (Supplementary Table S5). The c.494C > A variant in CLRN2 replaces a polar uncharged amino acid (threonine) with a positively charged amino acid (lysine) in clarin 2, (p.(Thr165Lys)) (Creixell et al. 2012). This variant, as well as homozygous loss-of-function alleles, are absent in population frequency databases. This suggests $C L R N 2$ is intolerant to biallelic loss-of-function. Our in-house collection of 89,041 additional exomes/genomes, including a multiethnic cohort of 842 exomes from probands with autosomal recessive hearing loss, identified four individuals from three families of Iranian, Turkish, and Emirati ethnicities, who carried the $C L R N 2 \mathrm{c} .494 \mathrm{C}>\mathrm{A}$ variant (allele frequency $2.24 \times 10^{-5}$ ). An Iranian hearing impaired individual was included among the carriers.

The c.494C > A variant involves the exchange of a novel polar threonine (Thr) residue to a basic lysine (Lys) amino acid that affects a highly conserved amino acid in the alpha-helix of the PMP-22/EMP/MP20/Claudin superfamily domain (Fig. 2a-c). Among clarin proteins, clarin 2 and clarin 1 show $34.9 \%$ identity with 81 identical and 91 similar amino acids (using UniProt (UniProt Consortium 2018), Fig. 2b). The outcome of CLRN1 pathogenic or likely pathogenic missense variants, as well as nonsense variants (queried from the Deafness Variation Database v8.2 (Azaiez et al. 2018)) are marked in red (Fig. 2b) along with the clarin 2 p.(Thr165Lys) amino acid substitution (Fig. 2b, asterisk). Interestingly, nine out of the 19 clarin 1 amino acid mutated residues are identical in clarin 2 . Three clarin 1 amino acid substitutions (p.(Leu163Pro), p.(Leu167Trp), and p.(Ile181Asn), NP_001182723.1) align in close proximity to the clarin 2 p.(Thr165Lys). Furthermore, clarin 1 p.Leu163Pro (Fields et al. 2002) and p.Ile181Asn (GarcíaGarcía et al. 2012), that are both reported in USH3A, are p.Leu150 and p.Ile168 in clarin 2. Most importantly, the threonine residue at position 165 (Thr165) CLRN2 is conserved across species and the corresponding amino acid in clarin 1 is a serine residue (Fig. 2a-b), a scenario often 
A The tetraspan-like clarin proteins
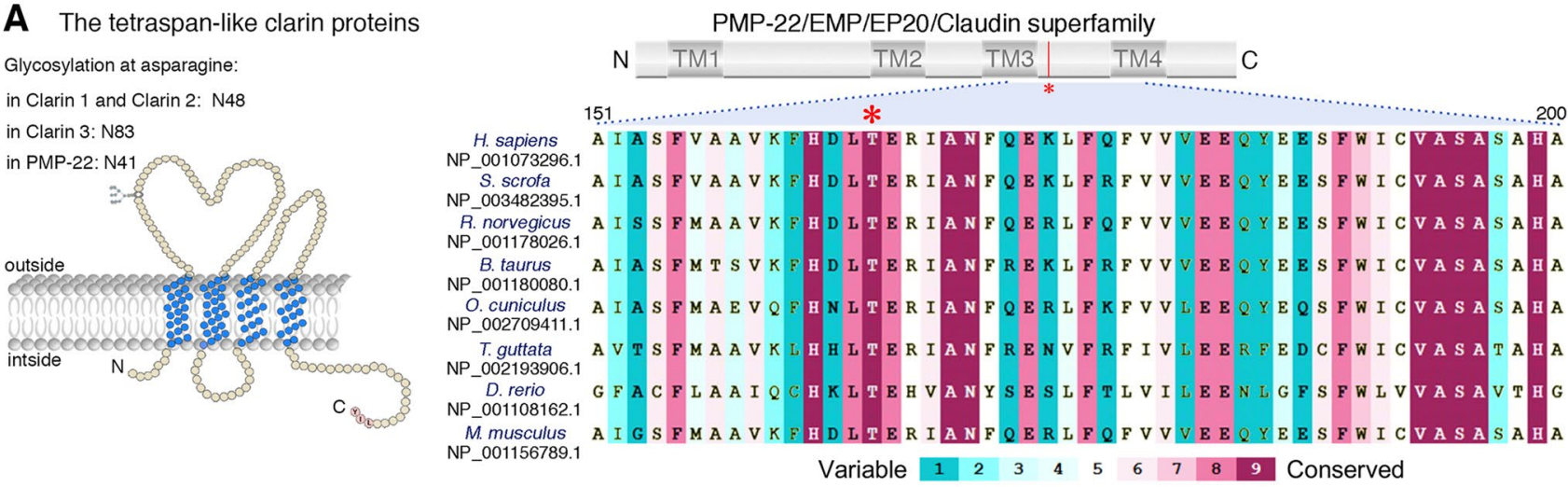

B CLRN2 H. sapiens (aa 1-232; SPIAOPK11) CLRN1 H. sapiens (aa 1-232; SP/P58418)

conservation

1 MPGWF KKAWYGLASLLSFSSF I L I IVALVVPHWLSGKILCQTGVDLVNATDRELVKF IGD 60 1 MPSQQKKII F OMAGVF SFACALGVVTALG TPLWIKATVLCKTGALLVWASGQELDKFMGE 60

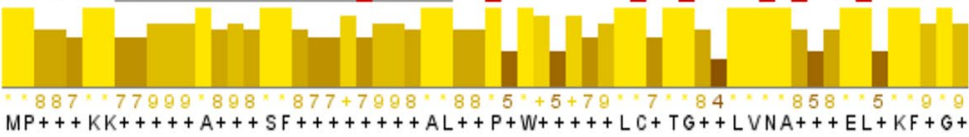

$\begin{array}{lll}C L R N 2 & H . & \text { sapiens (aa 1-232; SP/AOPK11) } \\ \text { CLRN1 } & H \text { sapiens (aa 1-232; SP/P58418) }\end{array}$

CLRN1 H. sapiens (aa 1-232; SP/P58418)

conservation

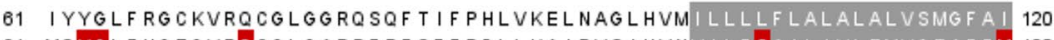
61 MQYGLFHGEGVRQCGLGARPFRFSFFPDLLKAIPVSIHVNVILFSAILIVLTMVGTAFFM 120

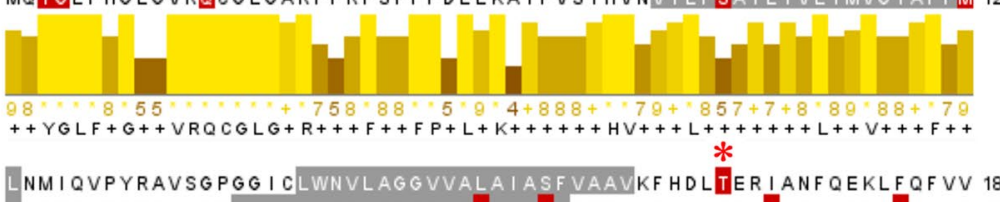

CLRN2 H. sapiens (aa 1-232; SP/AOPK11) 121 CLRN1 H. sapiens (aa 1-232; SP/P58418) 12

conservation
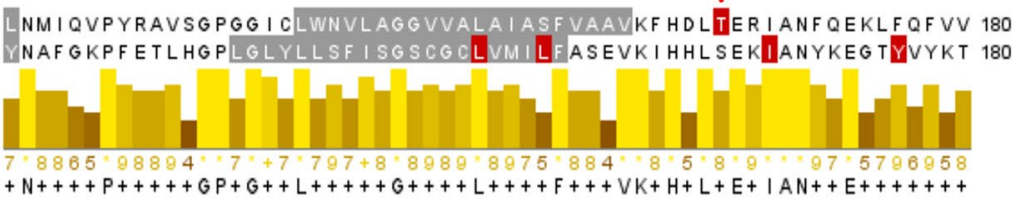

$\begin{array}{lll}\text { CLRN2 } & H . \text { sapiens (aa 1-232; SP/AOPK11) } \\ \text { CLRN1 } & H \text {. sapiens (aa 1-232; SP/P58418) }\end{array}$

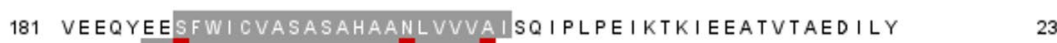

CLRN1 H. sapiens (aa 1-232; SP/P58418)

conservation

C Clarin 2 (p.Thr165): wild type

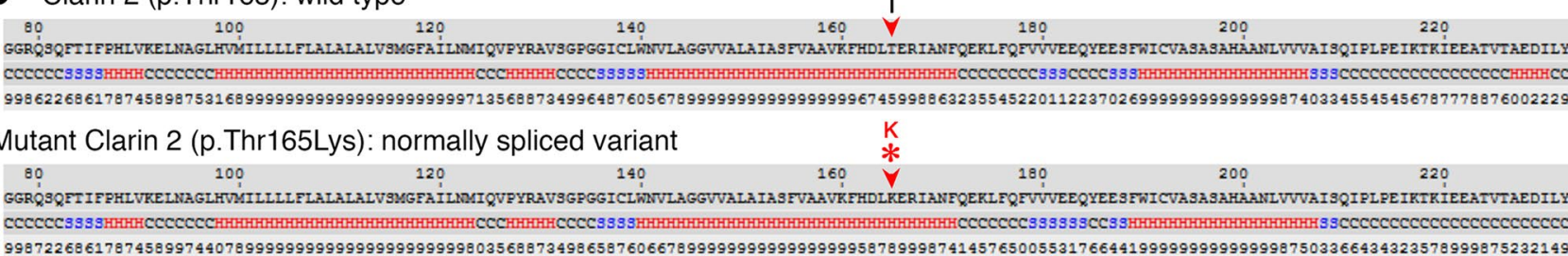

Fig. 2 Conservation of the p.Thr165 residue, and clarin 1/clarin 2 alignment. a Overview of clarin 2 protein and modular structure of the PMP-22/EMP/EP20/Claudin superfamily, with amino acid residue coordinates and position of the p.(Thr165Lys) substitution shown (upper panel). An alignment of the amino acid sequences from the segment of clarin 2 (represented by dashed lines) from vertebrate species shows the Thr165 position (asterisk) is well conserved among vertebrates. b Alignment of clarin 2 (UniProtKB: A0PK11, upper alignment) and clarin 1 (UniProtKB: P58418, lower align-

associated with conserved phosphorylation site residue, here by serine/threonine protein kinases (Creixell et al. 2012).

In addition to causing an amino acid missense substitution, computational analysis also predicts that the c. 494C > A variant will create an exonic splicing enhancer ment) amino acid residues. Transmembrane domains are marked in grey, conservation is shown in yellow, and consensus sequences are shown below for the 232 amino acid proteins. Missense and nonsense variants in clarin 1 (Deafness Variation Database v8.2) and clarin 2 (present study, asterisk) are marked in red. c The predicted secondary structure of human clarin 2 (NP_001073296.1) wild-type (Thr165) and mutated (Thr165Lys) protein. H represents alpha-helix, S represents beta-strand and $\mathrm{C}$ represents coil

(ESE) motif, modifying the ESE hexameric sequence landscape of exon 3, which could interfere with the normal processing of CLRN2 mRNA (Figs. S2a-b; ESEfinder and RESCUE-ESE, Human Splicing Finder) (Cartegni et al. 2003; Desmet et al. 2009; Fairbrother et al. 2004). To investigate 
the effect of the c.494C > A variant on CLRN2 splicing, we used mini-gene assays using two different exon-trapping vectors and three different cell lines, Cos-7, ARPE-19, and HEK 293 T. The mini-gene contained the 3 ' end of intron 2, all of exon 3 (with and without the CLRN2 variant), and $\sim 50 \mathrm{bp}$ of the 3' UTR (Fig. 3a) and was transfected into COS-7 and ARPE-19 cells. As a negative CLRN2 control, we used the rs 117875715 SNP, a common polymorphism, with a global MAF of $\sim 1.25 \%$ and $>100$ homozygous alleles reported in
gnomAD (Lek et al. 2016) (http://gnomad.broadinstitute.org/ variant/4-17528480-G-A) that is 20 nucleotides away from c. $494 \mathrm{C}>$ A. Given its frequency, rs 117875715 is predicted to be benign for hearing loss. Of note, this polymorphism is absent in the proband and family members reported here. Since exon 3 is the last exon of CLRN2, we designed our PCR primers to exclude the human poly-A signal and used the poly-A signal native to the pET01 vector. As expected for WT CLRN2 (c.494C), we detected the splicing of the 5'

\section{A CLRN2 allele and mini-gene splice construct design}

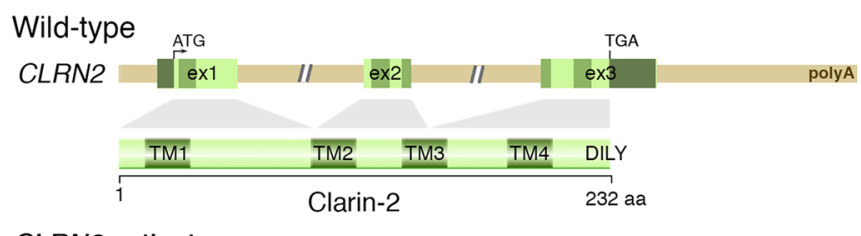

CLRN2 patients

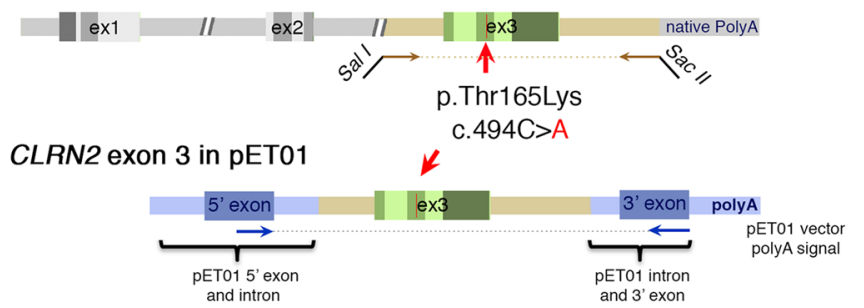

\section{B RT-PCR of transcripts from post-mini-gene transfected COS-7 cells}

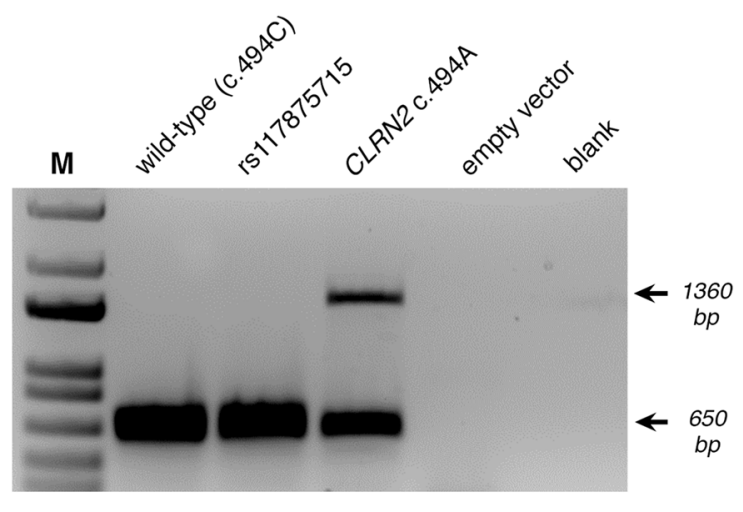

\section{Premature stop codon (TGA) in abnormally spliced CLRN2 (c.494A) variants}

Normally spliced

CLRN2 (c.494A)

$\&$ clarin 2 protein:

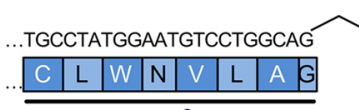

GCGGCGTCGTGGCGTTAGCCATC.. \begin{tabular}{|l|l|l|l|l|l|l|l|l|l|l|l|l|l|l|l|l|l|l|l|l|l|l|}
\hline $\mathrm{G}$ & $\mathrm{G}$ & $\mathrm{V}$ & $\mathrm{V}$ & $\mathrm{A}$ & $\mathrm{L}$ & $\mathrm{A}$ & $\mathrm{I}$ & $\mathrm{A}$ & $\mathrm{S}$ & $\mathrm{F}$ & $\mathrm{V}$ & $\mathrm{A}$ & $\mathrm{A}$ & $\mathrm{V}$ & $\mathrm{K}$ & $\mathrm{F}$ & $\mathrm{H}$ & $\mathrm{D}$ & $\mathrm{L}$ & $\mathrm{K}$ & $\mathrm{E}$ & $\mathrm{R}$ \\
\hline
\end{tabular}

Abnormally spliced

CLRN2 (c.494A)

$\&$ clarin 2 protein:

...TGCCTATGGAATGTCCTGGCAGGTAAGAAGTCCCTTAGGGAGAGAAAATATGTCCACACCATCATAGGGCTGGGCTCCAAGTAGTTGGGCAGAAGGTGTGA \begin{tabular}{|l|l|l|l|l|l|l|l|l|l|l|l|l|l|l|l|l|l|l|l|l|l|l|l|l|l|l|l|l|l|l|l|l|}
\hline C & L & W & N & V & L & A & G & K & K & S & L & R & E & R & K & L & C & P & H & H & H & R & A & G & L & Q & V & V & G & Q & K & V \\
\hline
\end{tabular}

exon $2 \ldots \ldots \ldots \ldots+\ldots \ldots+\ldots$

chr4:17,524,666-17,524,746 81 bp. enter position, gene symbol, HGVS or search terms $g 0$

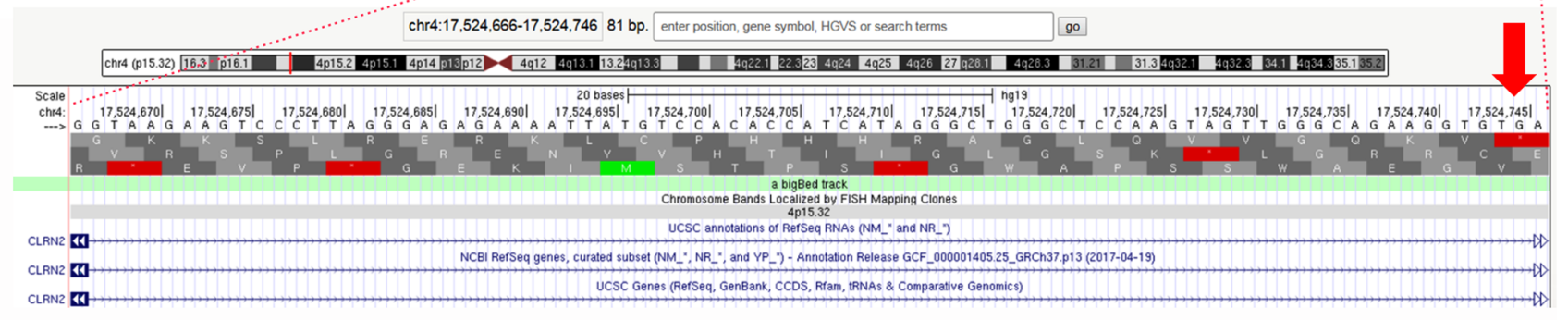

Fig. 3 Analysis of the $C L R N 2$ c. $494 \mathrm{C}>$ A variant on splicing. a Schematic illustration of the mini-gene splice construct design. Genomic representation of $C L R N 2$, including the position of the missense variant c.494C > A (arrow) on exon 3 with 3' UTR (green), and the $5^{\prime}$ UTR, as well as exons 1 and 2 (grey) (upper panel). Regions captured by mini-gene PCR primers are represented in brown. Schematic illustration of the mini-gene splice construct including exon 3 and its flanking sequence (green) cloned into multiple cloning sites (SalI and SacII sites) of pET01 backbone vector (lower panel). Blue boxes represent native exons of the pET01 vector. b RT-PCR of transcripts from post-mini-gene transfected COS-7 cells. Amplicons derived from the transcripts of WT (CLRN2), a benign CLRN2 polymor- phism (rs117875715, chr4(GRCh37):g.17,528,480G >A), the CLRN2 c. $494 \mathrm{C}>\mathrm{A}$ variant and a negative control, were visualized on a $1.5 \%$ agarose gel. The SNP, rs117875715, was used to test and validate the designed WT and mutant mini-gene assay. The $\sim 650 \mathrm{bp}$ amplicon was associated with the WT and validation control rs117875715. The amplicon derived from the CLRN2 c.494C > A transcripts showed two bands: a 650 bp band and a larger $\sim 1360$ bp band, indicating retention of intron separating the donor site of the $5^{\prime}$ exon and the acceptor site of CLRN2 exon 3. c Retention of intron in CLRN2 c.494C > A mini-gene results in a stop codon (TGA) after CLRN2 exon 2 
native pET01 exon only to exon 3 of $C L R N 2$ (Fig. 3a-b). The same normal splicing was obtained in all cell types transfected with CLRN2 containing the control (rs117875715) variant (Fig. 3b). However, the c. $494 \mathrm{C}>\mathrm{A}$ variant yielded two bands; one $\sim 650$ bp band matching the expected normally spliced exon, and a second abnormal band that was approximately $\sim 1360 \mathrm{bp}$ (Fig. 3b). Sequencing of these amplicons validated normal splicing including the c.494A variant and also revealed a retained intron 2 in the aberrantly spliced transcript (Supplementary Fig. S3c). The retention of intron 2 results in a new reading frame that introduces a stop codon 26 amino acids after the native exon 2 splice site (p.(Gly146Lysfs*26)) (Fig. 3c). These results were replicated using the pSPL3b vector and HEK $293 \mathrm{~T}$ cells (Supplementary Fig. S3a-c), confirming the c.494C $>$ A induced normal and aberrant splicing, independent of the cell type context. Following TA-cloning of cDNA amplicons from the homozygous individual (from Supplementary Fig. S3b), 23 of 26 amplicons (88.5\%) showed normal splicing, and 3 of 26 amplicons (11.5\%) showed a retained intron.

The mini-gene splicing assays and sequence analyses clearly show that the c.494C $>$ A affects a highly conserved and key residue in clarin 2 sequence, while also creating aberrant mRNA splicing in vivo likely leading to a truncated protein. Altogether, this further confirms that variants in $C L R N 2$ can lead to sensorineural hearing loss.

\section{Clrn2, a hair cell expressed gene key to hearing also in zebrafish and mice}

To further study the role of clarin 2 in the inner ear, we investigated its expression and analyzed potential impact of Clrn2 loss-of-function in two other species, zebrafish and mice.

\section{clrn2 in zebrafish}

Taking advantage of larva transparency, we used zebrafish as a model to investigate the clarin 2 expression during early embryonic development. The RT-qPCR at different developmental stages revealed that clrn2 mRNA was first detected at $18 \mathrm{hpf}$ (Fig. 4a), a stage when the otic placode begins to form the otic vesicle in zebrafish (this stage is similar to mouse embryonic day 9 (E9), a stage of otic placode formation) (Kopecky et al. 2012; Whitfield et al. 2002). clrn2 mRNA expression increased (twofold at 72 and $96 \mathrm{hpf}$ compared to $18 \mathrm{hpf}$ ) and was maintained at later stages, up to $120 \mathrm{hpf}$ (Fig. 4a). Comparative analyses of clrn 2 mRNA expression in different adult tissues of zebrafish revealed a significant enrichment in utricle, saccule and lagena of the inner ear (Fig. 4b). Our data are in agreement with RNA expression data from the Genotype-Tissue Expression (GTEx) project, wherein CLRN2 mRNA in humans is enriched in the nervous system, testis, kidney, salivary gland, and lung. CLRN1 has a similar expression profile in humans.

To determine clrn2 cellular expression, we used WISH in the inner ear of 3 and $5 \mathrm{dpf}$ embryos (Fig. $4 \mathrm{c}-\mathrm{d}$ ). Unlike the $c l r n 2$ sense probe, the anti-sense clrn2 revealed strong expression in the otic vesicle, similar to the expression of anti-sense pvalb9, used as a marker of hair cells (Fig. 4c). Histological examination of $5 \mathrm{dpf}$ embryos further confirmed that $\operatorname{clrn} 2$ expression is more specifically, restricted to hair cells, and is not expressed in the supporting cells of the inner ear (Fig. 4d).

To elucidate the function of $c l r n 2$ in zebrafish, we used CRISPR/Cas9 to generate loss-of-function alleles. To maximize the knockout efficiency, we used five sgRNAs targeting the first and second exon of clrn2 gene (Supplementary Fig. S4). Injected embryos (crispants) were sequenced and, as expected, a mix of alleles in the form of deletions ranging from 4 to $73 \mathrm{bp}$, as well as insertions spanning +1 to $+11 \mathrm{bp}$ were observed. The majority of the variants were frameshift that would most likely create a premature stop codon in the protein (Supplementary Fig. S4). The RT-qPCR analyses on injected embryos showed that clrn 2 crispants have a significantly reduced amount of clrn 2 mRNA (Fig. 4e), suggesting nonsense mediated decay, leading to disrupted clarin 2 protein function.

To test acoustic responses, we performed the AEBR analysis. The clrn 2 crispants showed significantly reduced response after sound stimulation (Fig. 4f) compared to the control animals, indicating a hearing loss phenotype. Considering clrn2 expression in hair cells (Fig. 4d), we investigated the architecture of their mechanosensory hair cell bundles, which are important for hearing and balance function in zebrafish. Interestingly, fluorescent phalloidin staining of the hair bundles of the inner ear in clrn 2 crispants $(n=10)$ showed disrupted hair bundle structure and fewer hair cells compared to the WT controls (arrowheads in Fig. 4g). This defective phenotype, suggesting a critical role in hair bundle structures, is similar to the hair bundles in zebrafish clrn 1 knockouts (Gopal et al. 2015), the orbiter mutants (defective in protocadherin 15 ( $p c d h 15)$, a gene associated with human Usher syndrome 1F) (Seiler et al. 2005) and ushlc morphants and ushlc mutants (Phillips et al. 2011).

\section{Clrn2 in mice}

To further assess the requirement of clarin 2 for auditory function in mammals, and assess further its role in auditory hair bundles, we extended our analyses to mouse. Consistent with expression data in zebrafish (Fig. 4a, c-d), single cell RNA-seq data available to visualize on the gEAR portal (umgear.org) show that in the mouse cochlear epithelium at postnatal day 1 (P1) and P7, Clrn2 transcripts are almost exclusively detectable only in inner and outer 


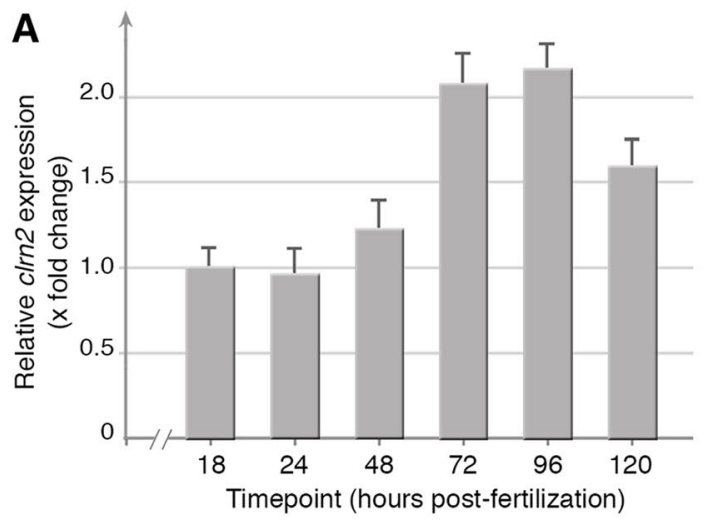

C clrn2 in the inner ear at 3 day post fertilization (dpf)

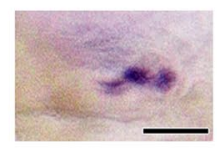

pvalb9 (anti-sense)

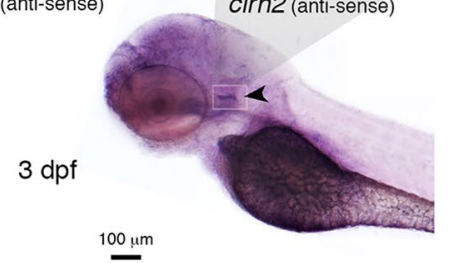

F AEBR in wild-type and clrn2 crispants

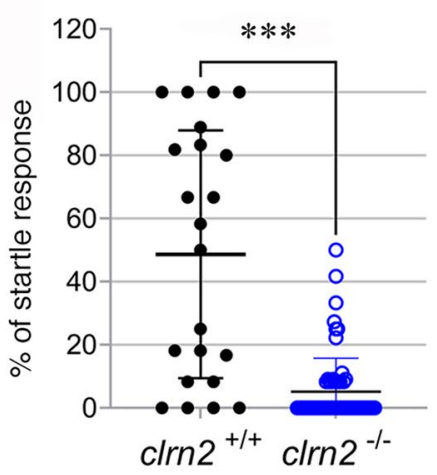

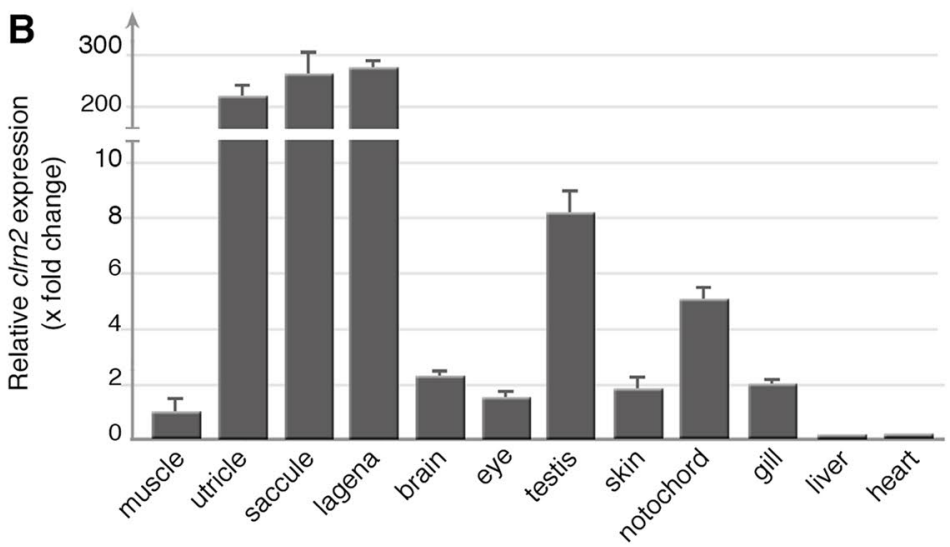

D clrn2 in the inner ear at $5 \mathrm{dpf}$

E Relative clrn2 expression ( $x$ fold change)

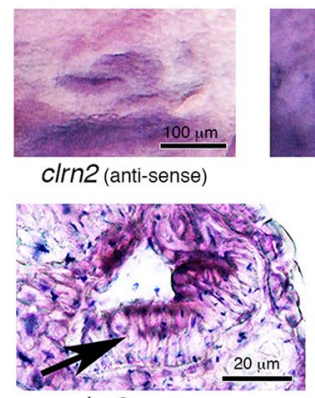

clrn2 (anti-sense)

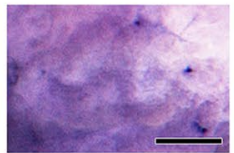

clrn2 (sense)

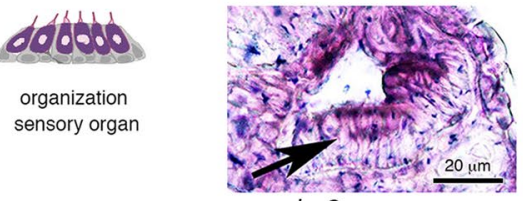

G

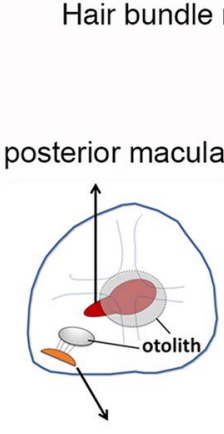

anterior macula

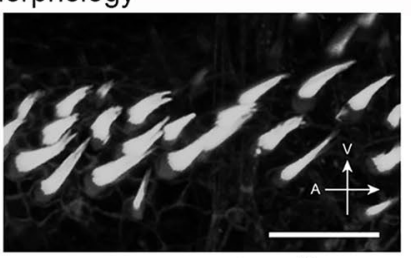

wild-type (clrn2 $\left.2^{+/+}\right)$

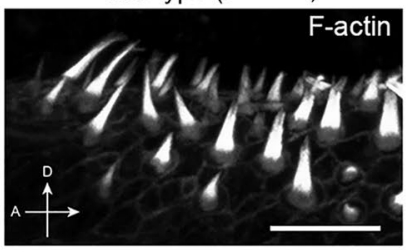

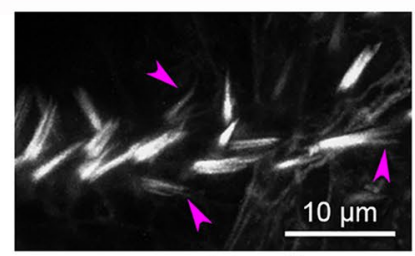

crispant $\left(c / r n 2^{-/-}\right)$

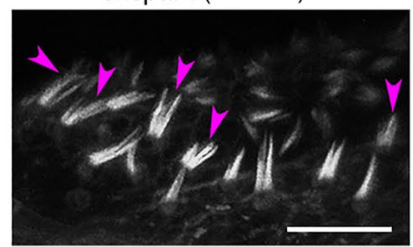

Fig. 4 Clarin 2 is required for the inner ear function in zebrafish. a RT-qPCR of clrn 2 mRNA expression from 1 to $120 \mathrm{hpf}$ of WT embryos/larvae. clarin 2 mRNA expression can be detected starting from $18 \mathrm{hpf}$ and then increased throughout development. Data shown are mean \pm SD and compared to $18 \mathrm{hpf}$. b RT-qPCR of clrn 2 mRNA expression in different adult tissues. Data shown are mean \pm SD and compared to muscle. c-d Whole-mount in situ hybridization (WISH) using antisense $\operatorname{clrn} 2$ probe reveals the inner ear expression of clrn 2 mRNA (relative dark purple color, black arrowhead) at 3 (c) and 5 (d) dpf embryos. Sense clrn2 probe was used as negative control and relative light purple color is considered as background. clrn2 mRNA was consistently expressed in hair cells within inner ear macula (c-d) with lined and arrayed structure. A known hair cell marker pvalb9 was used as an indicator for hair cells in the inner ear of 3

dpf embryos (c). Cryosection was performed after clrn2 WISH at 5 dpf to confirm the small patch of signal on the macula is from hair cells rather than supporting cells (d, black arrow lower panel). Scale bar $=100 \mu \mathrm{m}$, except lower panel in D $(20 \mu \mathrm{m})$. e RT-qPCR of clrn2 mRNA expression level was decreased $70 \%$ in clrn 2 crispants compared to uninjected larvae, indicating clrn 2 was successfully knocked out $(p=2.06 \mathrm{E}-06)$. Data shown are mean \pm SD. $* * * p<0.001$, twotailed unpaired Student's $t$ test. f Acoustically evoked behavioral responses (AEBR) in clrn2 wild type and crispants reveal significant reduction of sound induced responses. g Phalloidin staining on clrn2 crispants show that the hair cells in the inner ear anterior and posterior maculae display splayed, thin and split structures (purple arrowheads). A, anterior to the left. D, dorsal to the top. V, ventral to the top. Scale bar $=10 \mu \mathrm{m}$ 
hair cell populations (Kolla et al. 2020) (see also Supplementary Fig. S5). We utilized a CRISPR/Cas9-engineered Clrn 2 mouse mutant, in which exon 2 has been deleted $\left(C l r n 2^{\text {del629) }}\right.$ (Fig. 5a). While this deletion leaves exon 3 in-frame with exon 1, exon 2 encodes two of the transmembrane domains present in the tetraspan clarin 2 protein (lower panels Fig. 5a), and is therefore expected to severely affect protein function. In a preliminary work focused on clarinet mice, which display a nonsense Clrn2 mutation, p.(Trp4*), we showed that addition of the Clrn $2^{\text {del629 }}$ allele into the clarinet background, Clrn2 ${ }^{\text {clarinet/del629 }}$, was unable to complement the clarinet allele causing hearing loss in these mice, indicating $\mathrm{Clrn} 2^{\text {del629 }}$ is also a loss-of-function allele (Dunbar et al. 2019). Here, we provide the first morphofunctional characterization of $\mathrm{Clrn} 2^{\text {del629/del629 }}$ mice, which, unlike the previously reported clarinet mice, are congenic on the C57BL/6N background, and measured ABRs in P21 ( \pm 1 day) mice in response to click and tone-burst stimuli.

Analysis of ABR thresholds, which is the lowest sound stimulus required to elicit measurable activity in the auditory
A Clrn2 in wild type and mutant mice CIrn2 allele and mouse wild-type protein

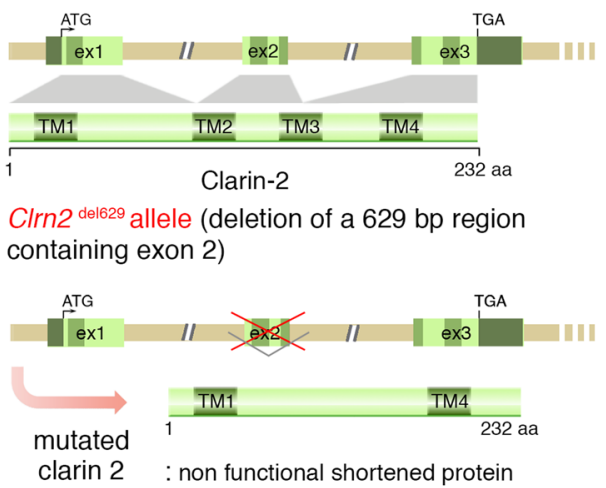

B ABR thresholds and DPOAEs in presence $(\square, \circ)$ or absence $(\square)$ of clarin 2

Clrn2 $2^{+/+} \circ$ Clrn2 del629/+ $\quad$ C Clrn2 del629/del629

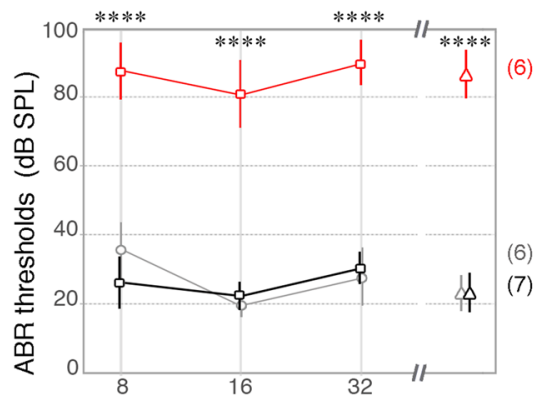

stimulus frequency $(\mathrm{kHz}) \quad$ click

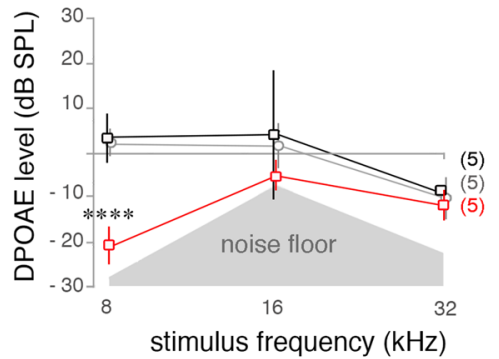

C Loss of short row stereocilia in CIrn2-deficient hair bundles

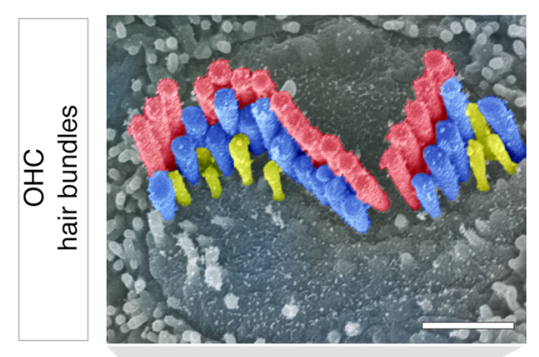

Clrn1 ${ }^{-1-}$

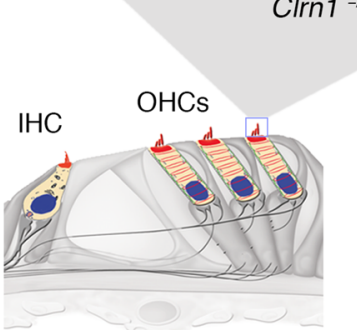

auditory sensory epithelium

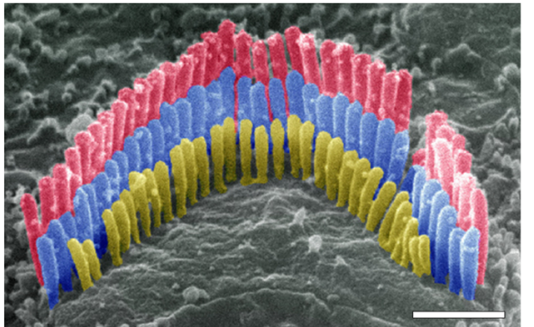

Clrn2 $2^{+/+}$
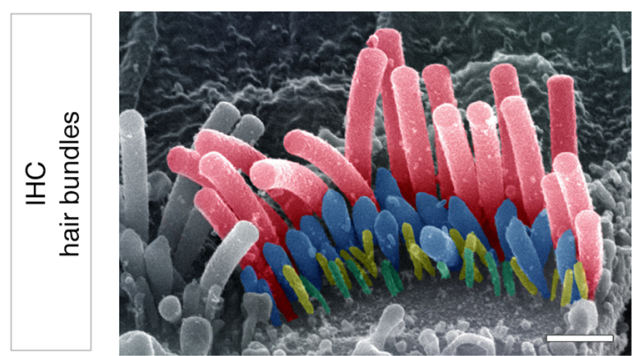

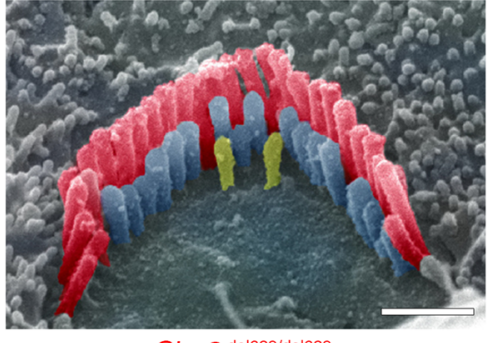

Clrn2 del629/del629

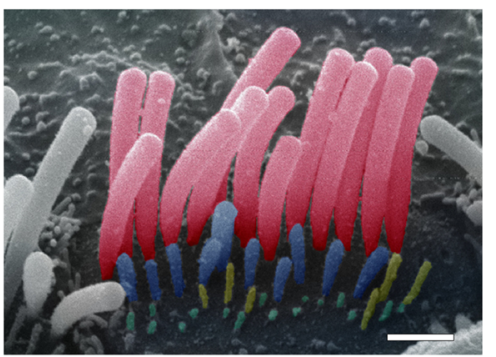

Fig. 5 Clarin 2 is required for hearing function in mouse. a The genomic structure of mouse Clrn2 (ENSMUST00000053250), and domains of the encoded tetraspan-like glycoprotein (232 amino acids). The positions of the transmembrane (TM) domains (dark green) and the structures of the WT Clrn2 and Clrn $2^{\text {del629 }}$ alleles are indicated. Deletion of exon 2 leads to a shortened clarin 2 lacking the two central transmembrane domains. b ABR threshold measurements at P21 $( \pm 1$ day $)$ show that Clrn$2^{\text {del629/del629 }}$ mice (red) exhibit a severe-to-profound hearing loss affecting all frequencies tested, with thresholds at $80 \mathrm{~dB}$ SPL and beyond. Age-matched Clrn2 $2^{+/+}$(black) and Clrn2 $2^{\text {del629/+ }}$ (grey) controls display thresholds within the expected range (15-40 dB SPL). Averaged DPOAE responses at P28 ( \pm 1 day), showing significantly reduced responses in Clrn2 del629/del629 mice. Data shown are mean \pm SD. $* * p<0.001$, oneway ANOVA. $\mathbf{c}$ Pseudo-colored scanning electron micrographs illustrate the three full rows, tallest (red), middle (blue) and short (yellow), of P28 ( \pm 1 day) stereocilia in IHC and $\mathrm{OHC}$ hair bundles. Unlike the fragmented hair bundle in $\mathrm{ClrnI}^{-/-}$mice, lack of clarin 2 does not affect the shape of IHC or OHC hair bundles. However, all the short row stereocilia have completely or partially regressed in the absence of either clarin protein. Scale bar $=1 \mu \mathrm{m}$ 
nerve, showed that homozygous $\left(\mathrm{Clrn} 2^{\text {del629/del629 }}\right)$ mice display very elevated thresholds ( $>80$ decibel sound pressure level (dB SPL)) at all frequencies tested: 8,16 and $32 \mathrm{kHz}$ (Fig. 5b). Whereas, Clrn $2^{\text {del629/+ }}$ mice exhibit thresholds comparable with those of WT $\left(\mathrm{Clrn}^{+/+}\right)$littermates ( $<40 \mathrm{~dB}$ SPL), demonstrating the absence of a heterozygous auditory phenotype (Fig. 5b).

To further assess cochlear function, DPOAEs were meas-

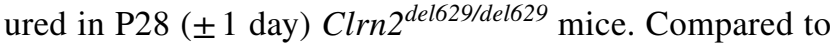
their $\mathrm{Clrn2}^{+/+}$and Clrn2 $2^{\text {del629/+ }}$ littermates, Clrn2 $2^{\text {del629/del629 }}$ mice have reduced DPOAEs (Fig. 5b) suggesting impaired outer hair cell (OHC) function.

To investigate stereocilia bundle morphology in Clrn $2^{\text {del629/del629 }}$ mice, we used scanning electron microscopy to examine the cochlear sensory epithelia. At P28 ( \pm 1 day), the inner and outer hair cell stereocilia bundles of Clrn2 mutant mice display the expected U- and V-shape, respectively, which contrasts with the grossly misshapen OHC bundles found in Clrn l mutant mice (Fig. 5c). However, while the patterning of the bundles appears normal in Clrn2 ${ }^{\text {del629/del629 }}$ mice the heights of their middle and short row stereocilia are visibly more variable compared with those of $\mathrm{Clrn}^{+/+}$littermates, and many of the short row 'mechanotransducing' stereocilia are missing (Fig. 5c).

Together, our findings establish that clarin 2 is key to hearing function in zebrafish and mouse, supporting that this protein has an evolutionary conserved role in the maintenance of hair bundle architecture in fish and mammals.

\section{Discussion}

We identify $C L R N 2$ as a novel deafness gene in human and zebrafish and describe a new deafness-causing allele in mice. Genetic study using gene mapping and exome sequencing of an extended Iranian family with multiple consanguineous marriages identified a pathogenic variant, c.494C $>$ A in exon 2 of $C L R N 2$ segregating with pre-lingual ARNSHL. Due to restricted expression of CLRN2 in accessible tissues such as blood or saliva, we performed in vitro splice analysis. The c. $494 \mathrm{C}>\mathrm{A}$ variant results in a missense and splicing defect in clarin 2. By producing mutant zebrafish and mice lacking clarin 2, we demonstrated the key role the protein plays to ensure normal structural and functional integrity of the hair bundle, the sound- and motion-receptive structure of inner ear hair cells.

The clarin gene family also includes the CLRN1 gene. Pathogenic variants in $C L R N 1$ have been linked to variable clinical outcomes, ranging from non-syndromic RP (Khan et al. 2011) to USH3A characterized by variable and progressive post-lingual hearing loss, RP, and variable vestibular responses (Plantinga et al. 2005). Several cases of later onset HL and/or RP, as late as the sixth decade of life, have been reported for USH3A patients (Ness et al. 2003). Clinical examination of affected individuals in this family, at the age of 25 (IV-8), 29 (IV-1), and 44 (IV-6) years of age, excluded the presence of additional syndromic features showing that homozygosity for the c.494C > A variant causes non-syndromic hearing loss, ranging from moderate-severe (IV-6) to profound (IV-1) deafness. In regard to the observed progressivity of the hearing impairment in clarinet mice (Dunbar et al. 2019), the earliest reported clinical diagnosis of hearing loss of the CLRN2 affected individuals in the family we present is between 2 and 3 years of age. Newborn hearing screening was not routinely performed when the affected individuals were born, so we cannot confirm hearing was normal at birth. In light of absent serial audiograms, we cannot report if the hearing loss experienced in these patients is progressive, as is observed in the mouse model (Dunbar et al. 2019). So far, we could not identify, through our current network, additional families with $C L R N 2$ variants. Featuring $C L R N 2$ as a new human deafness gene, future genetic screenings of hearing impaired families worldwide will probably unveil additional CLRN2 families and provide important clues about associated clinical phenotype progression and severity.

Our data showed that the CLRN2 c.494C > A variant probably affects protein function in two ways: (1) as a missense variant (p.(Thr165Lys)) producing a mutant full length protein and (2) as a splice variant leading to intron retention (Fig. 3b, and Supplementary Fig. S3b-c) expected to cause a premature stop codon 26 amino acids into intron 2 (p.(Gly146Lysfs*26)).

In normally spliced $C L R N 2$ transcripts, the c. $494 \mathrm{C}>\mathrm{A}$ variant affects an amino acid that is highly conserved among PMP-22/EMP/EP20/Claudin superfamily proteins. Two potential mechanisms could synergistically contribute to the disruptive effect of the missense variant. First, the replacement of threonine with lysine, an amino acid with a positively charged 'bulky' side chain (lysine), may affect protein folding (Creixell et al. 2012) and transport to the plasma membrane. Membrane proteins sort to the plasma membrane via the conventional secretory pathway associated with ER-to-Golgi complex (Viotti 2016). Misfolded membrane proteins are typically retained in the endoplasmic reticulum (ER) and degraded by the ER-associated degradation pathway (Kincaid and Cooper 2007; Sano and Reed 2013). It is possible that a small fraction of the misfolded clarin 2 p.(Thr165Lys) could reach the plasma membrane via the unconventional secretory pathway, similar to that reported for clarin 1 p.(Asn48Lys) (p.(N48K)) (Gopal et al. 2019). The unconventional secretory pathway is induced by the ER-associated misfolded or unfolded protein response (Kinseth et al. 2007; Schröder and Kaufman 2005). However, the mutant clarin 2 reaching the surface may be functionally inactive. Second, evolutionarily conserved threonine residues are also conserved protein phosphorylation 
sites. Phosphorylation adds a negative charge to the side chain of the amino acid and it serves as an important posttranslational mechanism for regulation of protein function (Pearlman et al. 2011). Loss of threonine at position 165 would potentially prevent functional activation of clarin 2 . As our attempts to discriminate impact of the amino acid substitution in a human cell line yielded no conclusive results (KNA, unpublished data), additional experiments in an in vivo context are essential to test these hypotheses and unravel the true pathogenic mechanism associated with the p.(Thr165Lys) missense variant.

With respect to the aberrantly spliced CLRN2 transcripts, variants that disrupt splicing machinery signals are recognized as significant contributors to human genetic diseases (Xiong et al. 2015), with variants shown to impact accurate recognition and removal of intronic sequences from pre-mRNA (Fairbrother et al. 2004). ESE sequences are cis-acting elements primarily recognized by the SR family proteins that function by recruiting core splicing machinery components to splice sites or by acting antagonistically against nearby silencing elements (Fairbrother et al. 2004; Graveley 2000; Kan and Green 1999). ESEs are often associated with introns that contain weak splicing signals, but they can also reside in exons and impact the splicing process. Using mini-gene assays in human cell lines, we could show that the CLRN2 variant-induced aberrant splicing amounts to $10-15 \%$, but its occurrence and rate in a biological context remains to be established. We, however, expect that the partial (only 3 out of 4 TM domains) and truncated (lack of $\mathrm{C}$-terminal region) protein resulting from aberrant splicing due to $C L R N 2$ c. $494 \mathrm{C}>\mathrm{A}$ variant is non-functional. Indeed, the integrity of all $4 \mathrm{TM}$ domains seems to be necessary for a functional clarin tetraspan protein. This is supported by our data in Clrn $2^{\text {del629/del629 mice }}$ where transcripts lacking exon 2 do exist, but if translated would result in a predicted protein lacking the two central transmembrane domains. The severe hearing loss observed in these $\mathrm{Clrn} 2^{\text {del629/del629 }}$ mice clearly indicate that such partial clarin protein is insufficient to ensure normal hearing.

Repeated attempts to detect endogenous clarin 2 in the inner ear, under various conditions of fixation and antigenretrieval at different postnatal stages were unsuccessful. We therefore used in situ hybridization in zebrafish and in silico analyses in mouse to confirm predominant expression of $C l r n 2$ in the sensory hair cells. To examine further the key role of clarin 2 in the inner ear, we generated zebrafish and mice lacking a functional protein. ABR measurements in $\mathrm{Clrn} 2^{\text {del629/del629 }}$ mice revealed an early-onset hearing loss with elevated hearing thresholds compared with their Clrn2 $2^{+/+}$littermate controls (mean click threshold $87 \mathrm{~dB}$ SPL \pm 7 s.d. and $24 \mathrm{~dB}$ SPL \pm 6 s.d., respectively). These data are consistent with early-onset hearing loss observed in another loss-of-function $\mathrm{Clrn} 2$ mutant ( $\left.\mathrm{Clrn} 2^{\text {clarinet }}\right)$, which harbors an early truncating nonsense variant (p.Trp4*)
(Dunbar et al. 2019). However, comparison with the previously reported Clrn $2^{\text {clarinet }} \mathrm{P} 21 \mathrm{ABR}$ data suggests that the extent of hearing loss in age-matched $\mathrm{Clrn} 2^{\text {del629 }}$ mice is more severe and less variable, which is most evident in the click ABR measures $(80 \pm 15 \mathrm{~dB}$ SPL and $87 \pm 7 \mathrm{~dB}$ SPL, respectively). This difference could be due to strain effect, with the $C l r n 2^{\text {clarinet }}$ mice being on a C57BL/6J background and the $C l r n 2^{\text {del629 }}$ mice being on, the related but distinct, C57BL/6N (Simon et al. 2013). Interestingly, startle response measurements in clrn 2 zebrafish crispants also reveal significant reduction in sound-induced responses. This, together with the severe-to-profound hearing loss already exhibited at P21 and the reduced DPOAEs in both Clrn2 $2^{\text {del629/del629 }}$ and in Clrn2 $2^{\text {clarinet/clarinet }}$ mice (Dunbar et al. 2019) points to gene defects likely affecting both inner hair cells (IHCs) and OHCs. This is further supported by scanning electron microscopy data showing loss of shortest row stereocilia in both the cochlear IHCs and OHCs. Phalloidin staining of clrn2 crispants also confirms hair bundle abnormalities in zebrafish. The loss of the mechanotransducing stereoclia, here the short stereocilia row in Clrn2 $2^{\text {del629/del629 }}$ and in $\mathrm{Clrn} 2^{\text {clarinet/clarinet }}$ mice is similar to that observed in hair cells defective for components of the mechanoelectrical transduction machinery. Electrophysiological recordings in OHCs of clarinet mice did indeed show that lack of clarin 2 causes significant reduction in hair cell mechanoelectrical transduction activity (Dunbar et al. 2019). However, whether the loss of short row stereocilia is a downstream consequence of defective MET activity or due to yet unknown structural changes in the stereocilia remains to be established.

In conclusion, we demonstrate the c. $494 \mathrm{C}>\mathrm{A}$ variant affects exon 3 splicing efficiency. We showed, for the first time, that $C L R N 2$ is a deafness-causing gene in humans. A variant causes hearing loss in humans, replicated by animal studies. Additional reports of families segregating CLRN2 biallelic variants will be crucial to refine and dissect the clinical course and characteristics of hearing loss due to this gene. Together, our studies in zebrafish and mice establish that hearing loss is probably due to defective protein in the hair cells, where the presence of clarin 2 is essential for normal organization and maintenance of the mechanosensitive hair bundles.

Acknowledgements We would like to extend our gratitude to the family for their participation. We thank Dr. Caroline Lekszas, Dr. Daniel Liedtke, and Dr. Indrajit Nanda from the Institute of Human Genetics at the University of Würzburg for their technical expertise. This work was supported by Intramural Funding (fortüne) at the University of Tübingen (2545-1-0 to B.V.), the Ministry of Science, Research and Art Baden-Württemberg (to B.V.), the Medical Research Council (MC_UP_1503/2 to M.R.B), ANR light4deaf (ANR-15-RHUS-0001), HearInNoise (ANR-17-CE16-0017), LHW-stiftung (to A.E.), and a grant from NIH/COBRE GM103636 (Project 3); the Presbyterian 
Health Foundation (PHF) Grant to G.K.V. This study was funded in part by NIDCDs R01s DC002842 and DC012049 to R.J.S and T32 GM007748 to K.T.B. L.A.D is a Medical Research Council DPhil student (1774724).

Author contributions $\mathrm{BV}, \mathrm{HA}, \mathrm{KTB}$, and AR conducted in vitro studies. SJL, LAD, SV, PV, CP, SVK, AE and MRB conducted in vivo studies. BF performed histology. BV, NM, RM, HA, KTB and HG performed genomic studies. NM, AS, GS, and HG identified patients, collected and analyzed clinical data, provided the biological specimens. RM, HA, KTB, FR, CB, DM, and HH conducted database analyses and data sharing. BV, RM, HA, KTB, KNA, RJHS, TH, AE, MRB and GKV wrote the manuscript. BV, HA, TH, RJHS, AE, MRB, GKV and HG supervised and conceived the study.

Funding Open Access funding enabled and organized by Projekt DEAL. This work was supported by Intramural Funding (fortüne) at the University of Tübingen (2545-1-0 to BV), the Ministry of Science, Research and Art Baden-Württemberg (to BV), the Medical Research Council (MC_UP_1503/2 to MRB), ANR light4deaf (ANR15-RHUS-0001), HearInNoise (ANR-17-CE16-0017), LHW-stiftung to AE), and a grant from NIH/COBRE GM103636 (Project 3); the Presbyterian Health Foundation (PHF) Grant to GKV. This study was funded in part by NIDCDs R01s DC002842 and DC012049 to RJS and T32 GM007748 to KTB. LAD is a Medical Research Council DPhil student (1774724).

Data availability All data needed to evaluate the conclusions in the paper are present in the paper and/or the Supplementary Materials. Additional data related to this paper may be requested from the authors.

\section{Compliance with ethical standards}

Conflicts of interest CB is an employee of Centogene AG (Rostock, Germany).

Ethical approval Human studies were approved by the Faculty of Medicine ethics commissions at the University of Würzburg (46/15) and Shahid Chamran University of Ahvaz (\#EE/97.24.3 17654). All zebrafish experiments were performed as per protocol (17-01) and approved by the Institutional Animal Care Committee of Oklahoma Medical Research Foundation (OMRF) (IACUC). Mouse studies at the MRC Harwell Institute were licenced by the Home Office under the Animals (Scientific Procedures) Act 1986, UK and additionally approved by the Institutional Animal Welfare and Ethical Review Body (AWERB).

Informed consent to participate Written informed consent was obtained from all participants.

Informed consent for publication Consent for publication was obtained from all participants.

Open Access This article is licensed under a Creative Commons Attribution 4.0 International License, which permits use, sharing, adaptation, distribution and reproduction in any medium or format, as long as you give appropriate credit to the original author(s) and the source, provide a link to the Creative Commons licence, and indicate if changes were made. The images or other third party material in this article are included in the article's Creative Commons licence, unless indicated otherwise in a credit line to the material. If material is not included in the article's Creative Commons licence and your intended use is not permitted by statutory regulation or exceeds the permitted use, you will need to obtain permission directly from the copyright holder. To view a copy of this licence, visit http://creativecommons.org/licenses/by/4.0/.

\section{References}

Abecasis GR, Cherny SS, Cookson WO, Cardon LR (2001) GRR: graphical representation of relationship errors. Bioinformatics 17:742-743. https://doi.org/10.1093/bioinformatics/17.8.742

Abecasis GR, Cherny SS, Cookson WO, Cardon LR (2002) Merlinrapid analysis of dense genetic maps using sparse gene flow trees. Nat Genet 30:97-101. https://doi.org/10.1038/ng786

Adato A et al (2002) USH3A transcripts encode clarin-1, a fourtransmembrane-domain protein with a possible role in sensory synapses. Eur J Hum Genet 10:339-350. https://doi. org/10.1038/sj.ejhg.5200831

Adzhubei IA et al (2010) A method and server for predicting damaging missense mutations. Nat Methods 7:248-249

Azaiez $\mathrm{H}$ et al (2018) Genomic landscape and mutational signatures of deafness-associated genes. Am J Hum Genet 103:484-497. https://doi.org/10.1016/j.ajhg.2018.08.006

Booth KT et al (2018a) Exonic mutations and exon skipping: Lessons learned from DFNA5. Hum Mutat 39:433-440. https://doi. org/10.1002/humu.23384

Booth KT, Kahrizi K, Najmabadi H, Azaiez H, Smith RJ (2018b) Old gene, new phenotype: splice-altering variants in CEACAM16 cause recessive non-syndromic hearing impairment. J Med Genet 55:555-560. https://doi.org/10.1136/jmedgenet-2018105349

Cartegni L, Wang J, Zhu Z, Zhang MQ, Krainer AR (2003) ESEfinder: a web resource to identify exonic splicing enhancers. Nucleic Acids Res 31:3568-3571

Chun S, Fay JC (2009) Identification of deleterious mutations within three human genomes. Genome Res 19:1553-1561. https://doi. org/10.1101/gr.092619.109

Creixell P, Schoof EM, Tan CS, Linding R (2012) Mutational properties of amino acid residues: implications for evolvability of phosphorylatable residues. Philos Trans R Soc Lond B Biol Sci 367:2584-2593. https://doi.org/10.1098/rstb.2012.0076

Cunningham CL, Müller U (2019) Molecular structure of the hair cell mechanoelectrical transduction complex. Cold Spring Harb Perspect Med 9:a033167

Desmet FO, Hamroun D, Lalande M, Collod-Beroud G, Claustres M, Beroud C (2009) Human splicing finder: an online bioinformatics tool to predict splicing signals. Nucleic Acids Res 37:e67. https://doi.org/10.1093/nar/gkp215

Doll J et al (2020) A novel missense variant in MYO3A is associated with autosomal dominant high-frequency hearing loss in a German family. Mol Genet Genomic Med. https://doi.org/10.1002/ $\operatorname{mgg} 3.1343$

Dulon D et al (2018) Clarin-1 gene transfer rescues auditory synaptopathy in model of Usher syndrome. J Clin Invest 128:33823401. https://doi.org/10.1172/JCI94351

Dunbar LA et al (2019) Clarin-2 is essential for hearing by maintaining stereocilia integrity and function. EMBO Mol Med 11:e10288

Fairbrother WG, Yeo GW, Yeh R, Goldstein P, Mawson M, Sharp PA, Burge CB (2004) RESCUE-ESE identifies candidate exonic splicing enhancers in vertebrate exons. Nucleic Acids Res 32:W187190. https://doi.org/10.1093/nar/gkh393

Fattahi $\mathrm{Z}$ et al (2019) Iranome: a catalogue of genomic variations in the Iranian population. Hum Mutat. https://doi.org/10.1002/ humu. 23880

Fields RR et al (2002) Usher syndrome type III: revised genomic structure of the USH3 gene and identification of novel mutations. Am J Hum Genet 71:607-617. https://doi.org/10.1086/342098 
García-García G et al (2012) Two novel disease-causing mutations in the CLRN1 gene in patients with Usher syndrome type 3. Mol Vis 18:3070-3078

Gillespie PG, Müller U (2009) Mechanotransduction by hair cells: models, molecules, and mechanisms. Cell 139:33-44. https:// doi.org/10.1016/j.cell.2009.09.010

Gopal SR et al (2015) Zebrafish models for the mechanosensory hair cell dysfunction in usher syndrome 3 reveal that clarin- 1 is an essential hair bundle protein. J Neurosci 35:10188-10201. https ://doi.org/10.1523/Jneurosci.1096-15.2015

Gopal SR, Lee YT, Stepanyan R, McDermott BM Jr, Alagramam KN (2019) Unconventional secretory pathway activation restores hair cell mechanotransduction in an USH3A model. Proc Natl Acad Sci U S A 116:11000-11009. https://doi.org/10.1073/ pnas. 1817500116

Graveley BR (2000) Sorting out the complexity of SR protein functions. RNA 6:1197-1211

Hofrichter MAH et al (2018) The conserved pArg 108 residue in S1PR2 (DFNB68) is fundamental for proper hearing: evidence from a consanguineous Iranian family. BMC Med Genet 19:81. https://doi.org/10.1186/s12881-018-0598-5

Hudspeth AJ (1997) How hearing happens. Neuron 19:947-950

Ingham NJ et al (2019) Mouse screen reveals multiple new genes underlying mouse and human hearing loss. PLoS Biol 17:e3000194. https://doi.org/10.1371/journal.pbio.3000194

Joensuu $T$ et al (2001) Mutations in a novel gene with transmembrane domains underlie Usher syndrome type 3. Am J Hum Genet 69:673-684. https://doi.org/10.1086/323610

Kan JLC, Green MR (1999) Pre-mRNA splicing of IgM exons M1 and M2 is directed by a juxtaposed splicing enhancer and inhibitor. Gene Dev 13:462-471

Kazmierczak P, Sakaguchi H, Tokita J, Wilson-Kubalek EM, Milligan RA, Muller U, Kachar B (2007) Cadherin 23 and protocadherin 15 interact to form tip-link filaments in sensory hair cells. Nature 449:87-91. https://doi.org/10.1038/nature06091

Khan MI et al (2011) CLRN1 mutations cause nonsyndromic retinitis pigmentosa. Ophthalmology 118:1444-1448. https://doi. org/10.1016/j.ophtha.2010.10.047

Kimmel CB, Ballard WW, Kimmel SR, Ullmann B, Schilling TF (1995) Stages of embryonic development of the zebrafish. Dev Dyn 203:253-310. https://doi.org/10.1002/aja.1002030302

Kincaid MM, Cooper AA (2007) Misfolded proteins traffic from the endoplasmic reticulum (ER) due to ER export signals. Mol Biol Cell 18:455-463. https://doi.org/10.1091/mbc.E06-08-0696

Kinseth MA, Anjard C, Fuller D, Guizzunti G, Loomis WF, Malhotra V (2007) The golgi-associated protein GRASP is required for unconventional protein secretion during development. Cell 130:524-534. https://doi.org/10.1016/j.cell.2007.06.029

Kircher M, Witten DM, Jain P, O'Roak BJ, Cooper GM, Shendure J (2014) A general framework for estimating the relative pathogenicity of human genetic variants. Nat Genet 46:310-315. https ://doi.org/10.1038/ng.2892

Kolla L et al (2020) Characterization of the development of the mouse cochlear epithelium at the single cell level. Nat Commun. https://doi.org/10.1038/s41467-020-16113-y

Kopecky B, Johnson S, Schmitz H, Santi P, Fritzsch B (2012) Scanning thin-sheet laser imaging microscopy elucidates details on mouse ear development. Dev Dyn 241:465-480. https://doi. org/10.1002/dvdy.23736

LaFave MC, Varshney GK, Vemulapalli M, Mullikin JC, Burgess SM (2014) A defined zebrafish line for high-throughput genetics and genomics: NHGRI-1. Genetics 198:167-170. https://doi. org/10.1534/genetics.114.166769

Lek M et al (2016) Analysis of protein-coding genetic variation in 60,706 humans. Nature 536:285-291. https://doi.org/10.1038/ nature 19057
Lekszas C et al (2020) Biallelic TANGO1 mutations cause a novel syndromal disease due to hampered cellular collagen secretion. Elife 9:e51319

Livak KJ, Schmittgen TD (2001) Analysis of relative gene expression data using real-time quantitative PCR and the 2(T)(-Delta Delta C) method. Methods 25:402-408. https://doi.org/10.1006/ meth.2001.1262

Mazzoli M, Van Camp G, Newton V, Giarbini N, Declau F, Parving A (2003) Recommendations for the description of genetic and audiological data for families with nonsyndromic hereditary hearing impairment. Audiol Med 1:148-150

Morton CC, Nance WE (2006) Newborn hearing screening-a silent revolution. N Engl J Med 354:2151-2164. https://doi. org/10.1056/NEJMra050700

Ness SL et al (2003) Genetic homogeneity and phenotypic variability among Ashkenazi Jews with Usher syndrome type III. J Med Genet 40:767-772

Ng PC, Henikoff S (2001) Predicting deleterious amino acid substitutions. Genome Res 11:863-874. https://doi.org/10.1101/gr.176601

O'Connell JR, Weeks DE (1998) PedCheck: a program for identification of genotype incompatibilities in linkage analysis. Am J Hum Genet 63:259-266. https://doi.org/10.1086/301904

Oza AM et al (2018) Expert specification of the ACMG/AMP variant interpretation guidelines for genetic hearing loss. Hum Mutat 39:1593-1613. https://doi.org/10.1002/humu.23630

Pearlman SM, Serber Z, Ferrell JE Jr (2011) A mechanism for the evolution of phosphorylation sites. Cell 147:934-946. https://doi. org/10.1016/j.cell.2011.08.052

Phillips JB et al (2011) Harmonin (Ush1c) is required in zebrafish Müller glial cells for photoreceptor synaptic development and function. Dis Model Mech 4:786-800

Plantinga RF, Kleemola L, Huygen PL, Joensuu T, Sankila EM, Pennings RJ, Cremers CW (2005) Serial audiometry and speech recognition findings in Finnish Usher syndrome type III patients. Audiol Neurootol 10:79-89. https://doi.org/10.1159/000083363

Rüschendorf F, Nürnberg P (2005) ALOHOMORA: a tool for linkage analysis using 10K SNP array data. Bioinformatics 21:2123-2125. https://doi.org/10.1093/bioinformatics/bti264

Sano R, Reed JC (2013) ER stress-induced cell death mechanisms. Biochim Biophys Acta 1833:3460-3470. https://doi.org/10.1016/j. bbamcr.2013.06.028

Schröder M, Kaufman RJ (2005) The mammalian unfolded protein response. Annu Rev Biochem 74:739-789. https://doi. org/10.1146/annurev.biochem.73.011303.074134

Schwarz JM, Cooper DN, Schuelke M, Seelow D (2014) MutationTaster2: mutation prediction for the deep-sequencing age. Nat Methods 11:361-362. https://doi.org/10.1038/nmeth.2890

Scott EM et al (2016) Characterization of Greater Middle Eastern genetic variation for enhanced disease gene discovery. Nat Genet 48:1071-1076. https://doi.org/10.1038/ng.3592

Seelow D, Schuelke M, Hildebrandt F, Nürnberg P (2009) HomozygosityMapper-an interactive approach to homozygosity mapping. Nucleic Acids Res 37:W593-W599. https://doi.org/10.1093/nar/ gkp369

Seiler C, Finger-Baier KC, Rinner O, Makhankov YV, Schwarz H, Neuhauss SC, Nicolson T (2005) Duplicated genes with split functions: independent roles of protocadherin 15 orthologues in zebrafish hearing and vision. Development 132:615-623. https:// doi.org/10.1242/dev.01591

Simon MM et al (2013) A comparative phenotypic and genomic analysis of C57BL/6J and C57BL/6N mouse strains. Genome Biol 14:R82. https://doi.org/10.1186/gb-2013-14-7-r82

Smith RJ, Bale JF Jr, White KR (2005) Sensorineural hearing loss in children. Lancet 365:879-890. https://doi.org/10.1016/S0140 $-6736(05) 71047-3$ 
Thisse C, Thisse B (2008) High-resolution in situ hybridization to whole-mount zebrafish embryos. Nat Protoc 3:59-69. https://doi. org/10.1038/nprot.2007.514

UniProt Consortium T (2018) UniProt: the universal protein knowledgebase. Nucleic Acids Res 46:2699. https://doi.org/10.1093/ nar/gky092

Varshney GK et al (2016) A high-throughput functional genomics workflow based on CRISPR/Cas9-mediated targeted mutagenesis in zebrafish. Nat Protoc 11:2357-2375. https://doi.org/10.1038/ nprot.2016.141

Viotti C (2016) ER to golgi-dependent protein secretion: the conventional pathway. Methods Mol Biol 1459:3-29. https://doi. org/10.1007/978-1-4939-3804-9_1

Waterhouse AM, Procter JB, Martin DM, Clamp M, Barton GJ (2009) Jalview Version 2-a multiple sequence alignment editor and analysis workbench. Bioinformatics 25:1189-1191. https://doi. org/10.1093/bioinformatics/btp033

Westerfield M (2000) The zebrafish book. A guide for the laboratory use of zebrafish (Danio rerio). 4th edn. University of Oregon Press, Eugene, Oregon, USA
Whitfield TT, Riley BB, Chiang MY, Phillips B (2002) Development of the zebrafish inner ear. Dev Dyn 223:427-458

Wright CF, FitzPatrick DR, Firth HV (2018) Paediatric genomics: diagnosing rare disease in children. Nat Rev Genet 19:253-268

Xiong HY et al (2015) RNA splicing. The human splicing code reveals new insights into the genetic determinants of disease. Science 347:125806

Yang J, Yan R, Roy A, Xu D, Poisson J, Zhang Y (2015) The I-TASSER Suite: protein structure and function prediction. Nat Methods 12:7-8. https://doi.org/10.1038/nmeth.3213

Publisher's Note Springer Nature remains neutral with regard to jurisdictional claims in published maps and institutional affiliations.

\section{Authors and Affiliations}

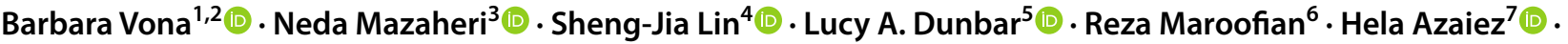

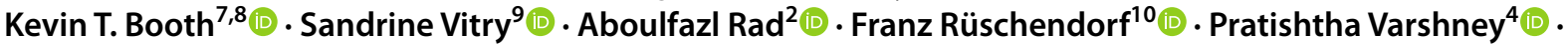 Ben Fowler ${ }^{11}$. Christian Beetz ${ }^{12}$ (1) . Kumar N. Alagramam ${ }^{13,14,15}$. David Murphy ${ }^{6}$ (1) Gholamreza Shariati ${ }^{16,17}$.

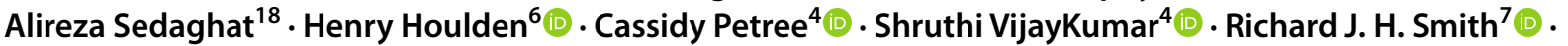

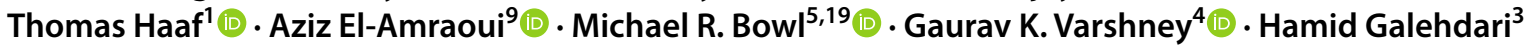

1 Institute of Human Genetics, Julius Maximilians University Würzburg, Würzburg, Germany

2 Department of Otolaryngology-Head and Neck Surgery, Tübingen Hearing Research Centre, Eberhard Karls University Tübingen, Tübingen, Germany

3 Department of Genetics, Faculty of Science, Shahid Chamran University of Ahvaz, Ahvaz, Iran

4 Genes \& Human Disease Research Program, Oklahoma Medical Research Foundation, Oklahoma City, OK, USA

5 Mammalian Genetics Unit, MRC Harwell Institute, Harwell Campus, Didcot OX11 0RD, UK

6 Department of Neuromuscular Disorders, UCL Queen Square Institute of Neurology, London WC1N 3BG, UK

7 Molecular Otolaryngology and Renal Research Laboratories, Department of Otolaryngology and Interdisciplinary Graduate Program in Molecular Medicine, Carver College of Medicine, University of Iowa, Iowa City, IA, USA

8 Department of Neurobiology, Harvard Medical School, Boston, MA, USA

9 Unit Progressive Sensory Disorders, Pathophysiology and Therapy Institut Pasteur, Institut de L'Audition, INSERM-UMRS1120, Sorbonne Université, 63 rue de Charenton, 75012 Paris, France

10 Max Delbrück Center for Molecular Medicine in the Helmholtz Association, 13125 Berlin, Germany
11 Imaging \& Histology Core, Oklahoma Medical Research Foundation, Oklahoma City, OK, USA

12 Centogene AG, Rostock, Germany

13 Department of Otolaryngology, School of Medicine, University Hospitals Cleveland Medical Center, Case Western Reserve University, 11100 Euclid Avenue, Cleveland, OH 44106, USA

14 Department of Neurosciences, Case Western Reserve University, 11100 Euclid Avenue, Cleveland, OH 44106, USA

15 Department of Genetics and Genomic Sciences, Case Western Reserve University, Cleveland, OH 44106, USA

16 Department of Medical Genetics, Faculty of Medicine, Ahvaz Jundishapur, University of Medical Sciences, Ahvaz, Iran

17 Narges Medical Genetics and Prenatal Diagnostics Laboratory, East Mihan Ave, Kianpars, Ahvaz, Iran

18 Diabetes Research Center, Health Research Institute, Ahvaz Jundishapur University of Medical Sciences, Ahvaz, Iran

19 UCL Ear Institute, University College London, 332 Gray's Inn Road, London WC1X 8EE, UK 